Ana Daniela Peres Rebelo
O Impacto de Inteligência Artificial na Criatividade de Vídeos

The Impact of Artificial Intelligence on the Creativity of Videos 
Ana Daniela Peres Rebelo
O Impacto de Inteligência Artificial na Criatividade de Vídeos

The Impact of Artificial Intelligence on the Creativity of Videos

Dissertação apresentada à Universidade de Aveiro para cumprimento dos requisitos necessários à obtenção do grau de Mestre em Comunicação Multimédia, realizada sob a orientação científica da Doutora Inês Maria Henriques Guedes de Oliveira, Professora auxiliar do Departamento de Comunicação e Arte da Universidade de Aveiro. 
Aos meus pais, que sempre me inspiraram e apoiaram. 


\section{o júri}

presidente

arguente principal

orientador
Prof. Doutor Mário Jorge Rodrigues Martins Vairinhos Professor Auxiliar, Universidade Aveiro

Prof a. Doutora Catarina Franco Lélis da Cruz Senior Lecturer, University of West London

Prof a . Doutora Inês Maria Henriques Guedes de Oliveira Professor auxiliar, Universidade de Aveiro 


\section{agradecimentos}

Quero expressar a minha gratidão às pessoas que me ajudaram e apoiaram durante a conceção desta dissertação.

Em primeiro lugar, gostaria de agradecer à minha orientadora, Inês Guedes, que me ajudou a concretizar esta dissertação, pela amabilidade, apoio e disponibilidade durante o projeto. Estou também grata à minha família por me fornecer as ferramentas que contribuíram para o meu desempenho durante este mestrado. Destaco o meu pai, por me inspirar e guiar durante os meus estudos.

Agradeço também ao Pedro Martins e ao Damion Verboom, pelo feedback deles e as suas contribuições para o estudo empírico. Finalmente, gostava de expressar a minha gratidão ao professor Nuno Barbosa, por disponibilizar as suas criações audiovisuais para este estudo. 


\section{palavras-chave}

resumo
Inteligência Artificial, Arte, Produto Artístico, Multimédia, Style Transfer, Criatividade

Neste estudo foi explorado o impacto do uso de um algoritmo de Inteligência Artificial (IA), Style Transfer, na avaliação dos elementos de criatividade de vídeos artísticos. O objetivo deste estudo foi verificar em que medida o uso deste sistema contribui para alterações na perceção qualitativa e quantitativa dos elementos de criatividade presentes nos vídeos, e verificar que mudanças ocorrem. Foi efetuado um estudo experimental, contemplando dois conjuntos de vídeos que foram visualizados por dois grupos: 1) um grupo de controlo ( $n=49$, dos quais 25 peritos e 24 não peritos); 2 ) um grupo experimental $(n=52$, dos quais 27 peritos e 25 não peritos). O primeiro conjunto, composto por seis vídeos sem transformação de IA (vídeos partilhados), foi exibido a ambos os grupos, visando a verificação da equivalência de critérios de avaliação. $O$ segundo conjunto, composto por seis vídeos (diferenciados) teve uma versão transformada por IA (exibida ao grupo experimental) e outra não transformada (exibida ao grupo de controlo). A cada participante foi solicitado que avaliasse, numa escala de Likert de cinco pontos, os vídeos, em seis elementos de criatividade e que caracterizasse a criatividade de cada vídeo com duas palavras. Os resultados quantitativos demonstraram equivalência de critérios nos vídeos partilhados entre o grupo experimental e o grupo de controlo (apenas uma de 36 comparações apresentou diferenças significativas). Relativamente à comparação quantitativa dos vídeos diferenciados (experimental versus controlo), 10 avaliações não apresentaram diferenças significativas, enquanto que cinco tiveram avaliações mais elevadas de criatividade no grupo experimental e cinco no grupo de controlo. Nas comparações qualitativas, em geral, a frequência dos termos usados pelos participantes de ambos os grupos foram semelhantes nos vídeos partilhados. Nos vídeos diferenciados ocorreram algumas diferenças. No seu conjunto, os resultados enfatizam a importância da mediação humana na aplicação de um algoritmo de Inteligência artificial na produção criativa, o que reforça a relevância do conceito de Inteligência Híbrida. 
keywords

\author{
abstract
}

Artificial Intelligence, Art, Artistic Product, Multimedia, Style Transfer, Creativity

In this study, the impact of using an Artificial Intelligence (AI) algorithm, Style Transfer, on the evaluation of the creative elements of artistic videos was explored. The aim of this study was to verify to what extent the use of this system contributes to changes in the qualitative and quantitative perception of the elements of creativity present in the videos, and to verify what changes occur. An experimental study was carried out, including two sets of videos that were watched by two groups: 1 ) a control group ( $n=49$, composed by 25 experts and 24 non-experts); 2 ) an experimental group ( $n=52$, composed by 27 experts and 25 non-experts). The first set, consisting of six videos without Al transformation (shared videos), was shown to both groups, aiming at verifying the equivalence of evaluation criteria. The second set, consisting of six videos (differentiated) with a version transformed by Al (displayed to the experimental group) and another untransformed (displayed to the control group). Each participant was asked to rate the videos, on a five-point Likert scale, on six elements of creativity and to characterize the creativity of each video with two words. The quantitative results showed equivalence of criteria in the shared videos between the experimental group and the control group (only one of 36 comparisons showed significant differences). Regarding quantitative comparisons of the differentiated (experimental versus control), 10 evaluations showed no significant differences, while five had higher evaluations of creativity in the experimental group and five in the control group. Concerning qualitative comparisons, in general, the frequency of terms used by participants in both groups was similar in the shared videos. In the differentiated videos there were some differences. Taken together, the results emphasize the importance of human mediation in the application of an Artificial Intelligence algorithm in creative production, which reinforces the relevance of the concept of Hybrid Intelligence. 


\section{Table of Contents}

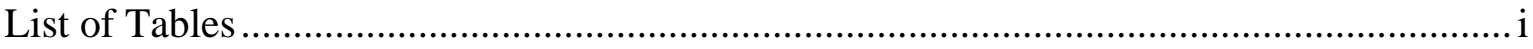

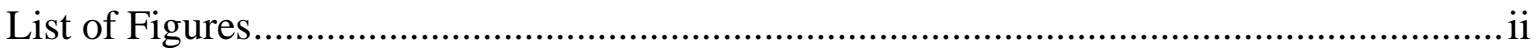

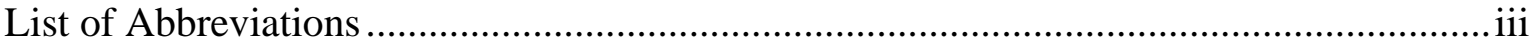

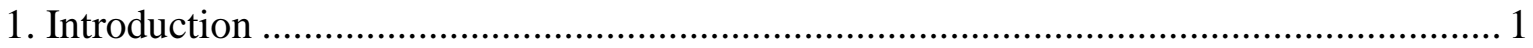

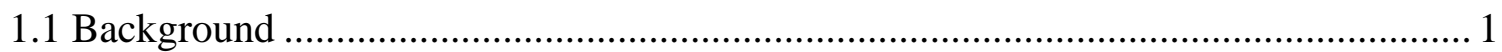

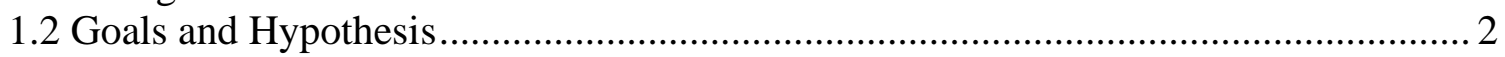

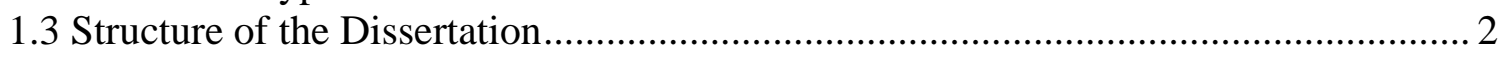

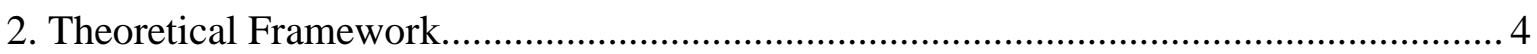

2.1 Defining and Conceptualizing Artificial Intelligence ........................................... 4

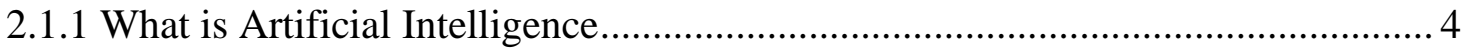

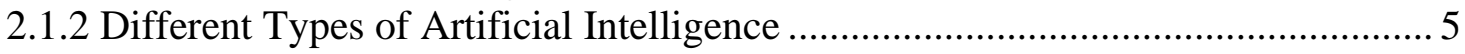

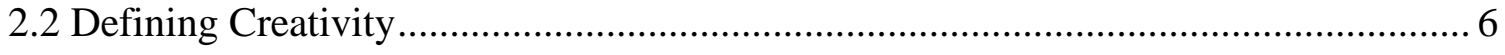

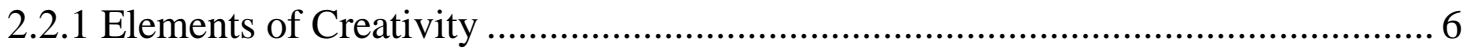

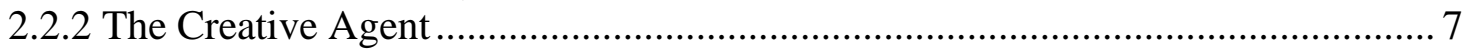

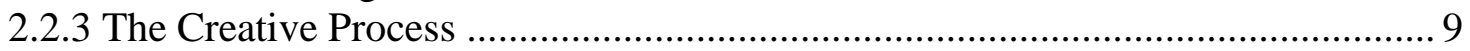

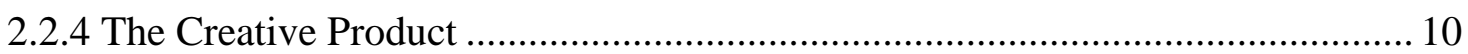

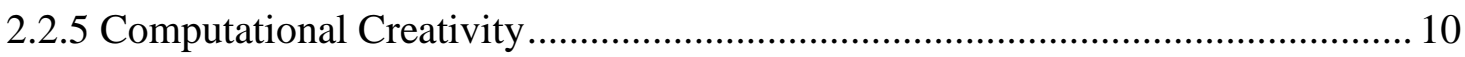

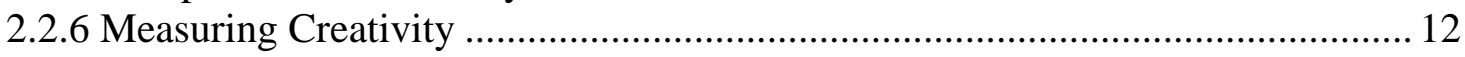

2.3 Artificial Intelligence Software and Algorithms for Creative Use .......................... 13

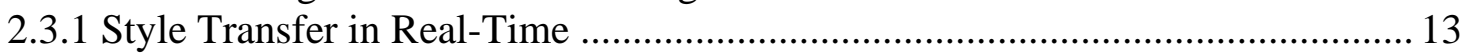

2.3.2 Style Transfer for Images through the use of Convolutional Neural Networks .. 14

2.3.3 An optimized version of Style Transfer through the use of Perpetual Losses.... 15

2.3.4 Optimization of Style Transfer through the use of Instance Normalization ........ 15

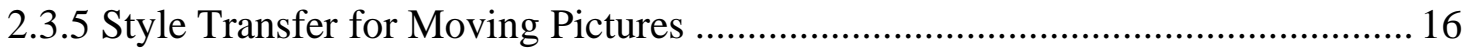

2.3.6 Creating Art Pieces Through the Use of Creative Adversarial Networks ........... 17

2.3.7 Artificial Intelligence in The Creation of Paintings........................................... 18

2.3.8 Other Relevant Artificial Intelligence Algorithms for Creative Production........ 19

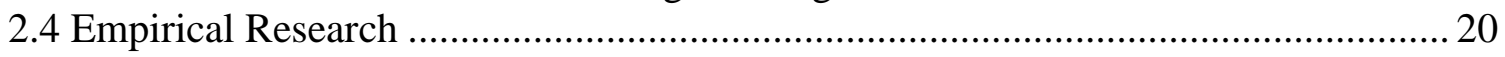

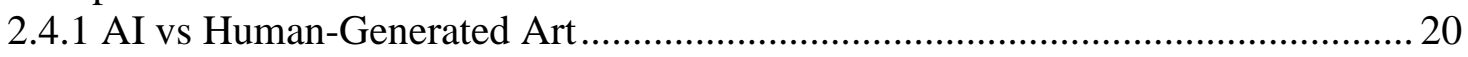

2.4.2 The Perception of the Identity of The Creative Agent........................................ 21

2.4.3 Artificial Intelligence Software for Ideation................................................. 22

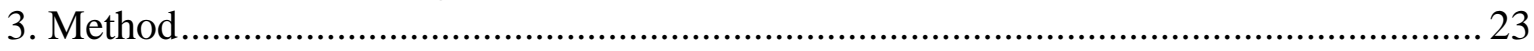

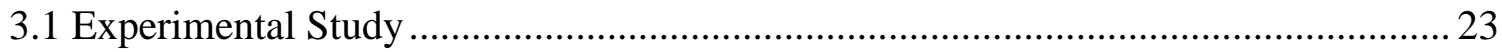

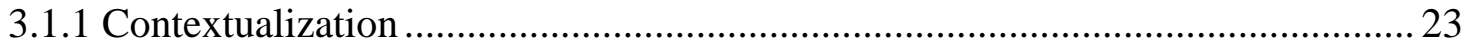

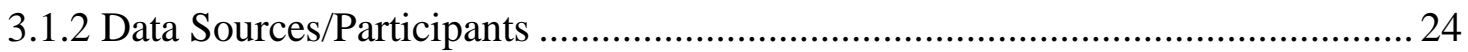

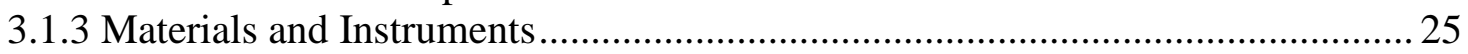

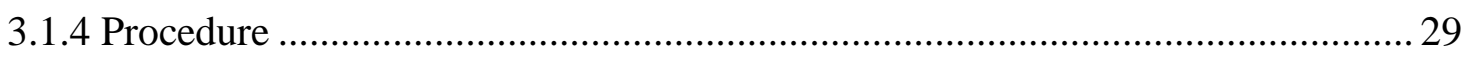

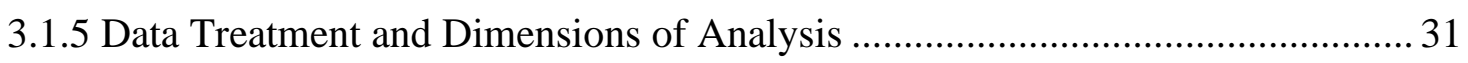

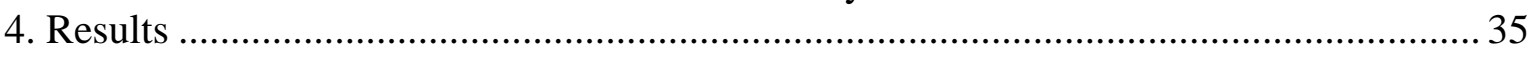

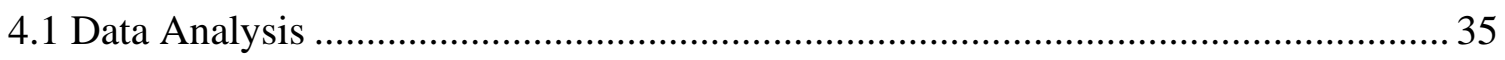

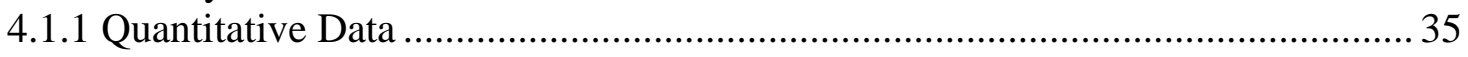

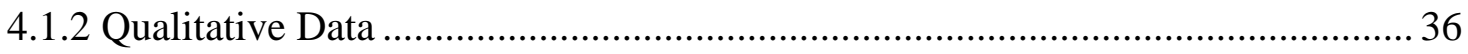

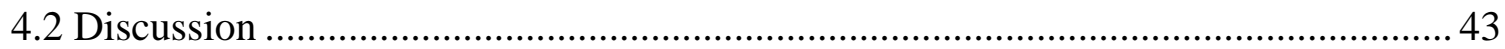

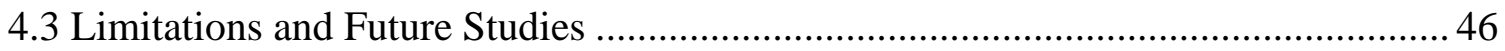

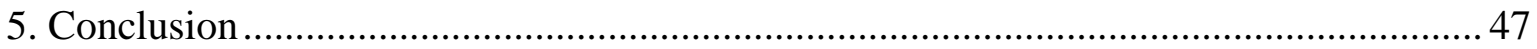

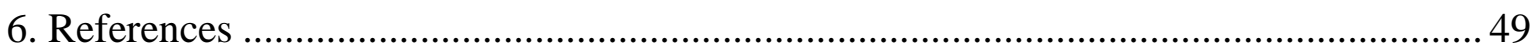

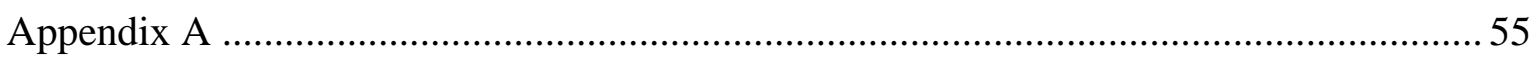

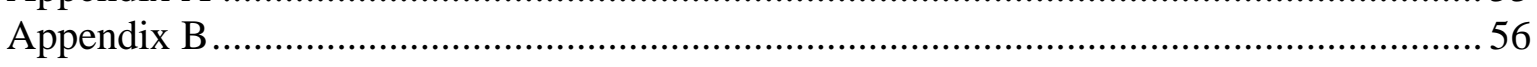

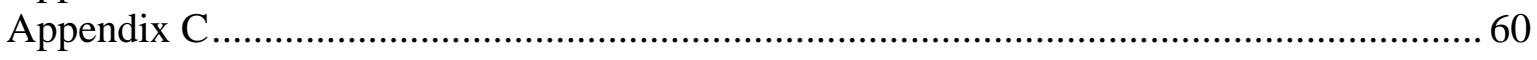




\section{List of Tables}

Table 1. Videos Used in the Questionnaire (Differentiated Videos) _.................... 27

Table 2. Videos Used in the Questionnaire (Shared Videos) _............................... 28

Table 3. Dimensions of Creativity 31

Table 4. Results Mann-Whitney U for each Differentiated Video

Table 5. Word Counts for Control and Experimental Groups _.......................... 39

Table 6. Qualitative Content per Video (Differentiated Videos) _................................ 40

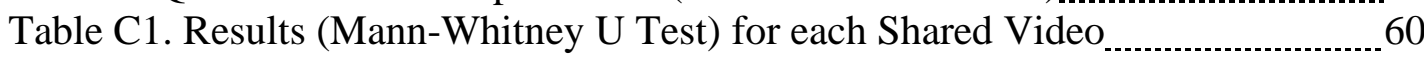




\section{List of Figures}

Figure 1. Pooling

Figure 2. CAN System

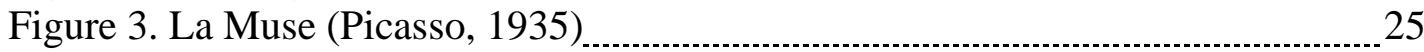

Figure 4. Undie (Picabia, 1913)

Figure 5. The Great Wave off Kanagawa (Hokusai, 1831) $\quad 26$

Figure 6. Word Cloud of The Shared Videos of the Control Group $\ldots \ldots \ldots \ldots . . . . . . . . . . . . . . . . . . . . . . . . . . .37$

Figure 7. Word Cloud of The Shared Videos of the Experimental Group _................38

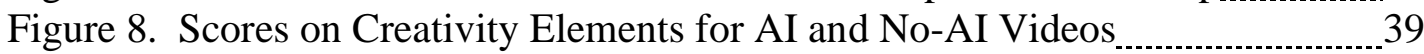




\section{List of Abbreviations}

AI - Artificial Intelligence

CAN - Creative Adversarial Networks

CAT - Consensual Assessment technique

CG - Control Group

CNN - Convolutional Neural Networks

CPSS - Creative Product Semantic Scale

EG - Experimental Group

GAN - Generative Adversarial Networks

ML - Machine Learning

NLP - Natural Language Processing

No-AI - No Artificial Intelligence 


\section{Introduction}

\subsection{Background}

Art, and its underlying creative process, has always existed in dynamic interaction with its environment and culture. Just like it was the case with the emergence of photography, it is believed that machine intelligence, also known as Artificial Intelligence (AI), will play a huge role in future artistic breakthroughs, deeply affecting the way art is perceived and created (Arcas, 2017). In "Art in the Age of the Machine Intelligence" (2017) Arcas defends that, just like it occurred with previous technologies, while some artists will embrace the medium, others will reject it. Leonel Moura, an artist that uses AI and Robotics for the creation of his artworks, argues that the most relevant aspect to have in consideration when a new process of art creation emerges is if it contributes to the development of the artistic area. The author speaks of the art movement Surrealism as an example to back up his argument, adding that it went as far as attempting, at a certain point, to remove the element of human consciousness from the creative process (Moura, 2018).

We already see a similar mechanism for art production in other technologies, a photographer works together with the machine in the creation of artworks, as such, it is not only working with his own biology but with a technological creation that consists of a machine with software. Arcas (2017) said he/she is already a "(...) hybrid artist, thinking, manipulating and encoding information with neurons in both the brain and the retina, working with muscles, motors, transistors, and millions of lines of code. Photographers are cyborgs" (p.6).

The author explains that while technologies like the photographic machine, attempt to mimic the human eye, AI software aims to do something distinct - to mimic the human brain. He adds that " $(. .$.$) machine intelligence is such a profoundly transformational$ technology; it is about creating the very stuff of thought and mind" (Arcas, 2017, p.7).

In the context of the development of Artificial Intelligence art, the question was raised by Audry and Ippolito (2019) - is AI the artist? Can computer programs be artists? Cohen, an artist who makes use of a program which he called Aaron, to create drawings for his artworks, argues that creativity does not exist alone in the software or inside the one who uses it for their creations, but it emerges from an exchange that occurs between both entities (Cohen, 2010).

There are multiple questions that can lead to future research on this subject. From those questions, we highlight as the most relevant for the present research the following: Firstly, studying to what extent AI can be more of an enabler that will help the artist create art (Lomas, 2018). Secondly, it is important to study the differences between artistic outputs made by human artists versus AI algorithms (Mazzone \& Elgammal, 2019). And thirdly, to study and deepen the understanding of the changes in the role of the artist and creative output that come from introducing AI in artistic creation (Arcas, 2017).

In this dissertation, we explore the changes in the perceived creativity of the produced artifacts through the use of the AI Style Transfer algorithm. This AI system works by analyzing the image of a determined style, separating it from the content, and later 
applying it to other imagery. It captures different characteristics of the input image and combines them to create a diverse collection of differently styled images (Gatys, Ecker \& Bethge, 2016).

\subsection{Goals and Hypothesis}

In the context of the cultural shift caused by the changes in the artistic paradigm driven by the use of the Artificial Intelligence systems presented above, the goal for this dissertation is defined as the analysis of the changes in the perceived creativity of videos produced by the application of an AI algorithm (Style Transfer) in comparison with the same videos without the AI transformation.

To achieve this, the following objectives were defined:

- To verify if the use of an Artificial Intelligence algorithm ensures a more creative artistic outcome in videos as perceived by individuals;

- To Identify what are the creative elements that the use of an Artificial Intelligence algorithm produces in an artistic product, the videos;

- To describe the perception by the spectators in regard to the pieces created with and without the use of an Artificial Intelligence algorithm.

\section{Hypothesis}

Based on the previously stated goals, the following general hypothesis was formulated for this study:

- The videos transformed through the use of an Artificial Intelligence algorithm (Style Transfer) are perceived as more creative in general than the ones without transformation.

\subsection{Structure of the Dissertation}

To achieve the objectives, the following structure was defined:

Firstly, an exploration of the definition and conceptualization of Artificial Intelligence is presented. Here, different types of machine learning techniques are introduced, such as supervised learning, unsupervised learning, reinforcement learning, and deep learning. In this section, the definitions of computer vision and imagery-based AI are also presented.

Secondly, creativity is defined and conceptualized through the presentation of different authors and their theories in regard to a series of topics, such as the elements of creativity, the creative agent, the creative process, the creative product, and computational creativity. Here, multiple ways of measuring creativity and their respective advantages and disadvantages are also introduced.

Next, different Artificial Intelligence systems for creative use are presented, including multiple Style Transfer algorithms that served as a source of inspiration for the creation of the one used in the empirical study of this dissertation. Other AI algorithms for creative production are also introduced, as well as some cases of artists that make use of AI algorithms in their work. This section is finalized through the presentation of some studies 
on Artificial Intelligence and Creativity that were relevant to the conceptualization of this research.

Regarding the empirical study section, the participants (experts and non-experts) are described, the sample size and the materials for data collection are presented, together with the reasons behind the choice of each video, with and without Style Transfer transformation. Experts were individuals with a university degree whose specialty is the field of visual arts. After this, the procedure is described. Finally, the results are presented and discussed, and some limitations of this research are highlighted. In this section, future studies that can overcome those limitations and contribute to the deepening of the knowledge on the subject are presented. 


\section{Theoretical Framework}

\subsection{Defining and Conceptualizing Artificial Intelligence}

In the context of this study, it is crucial to highlight the definition of Artificial Intelligence and the different branches of this area in order to further understand the functioning of the Style Transfer algorithm.

\subsubsection{What is Artificial Intelligence}

According to Wang (2009), intelligence consists of a movement towards the acquisition of competence and knowledge. It also manifests itself through the competence of problemsolving. The author stated that "it is a profound human wonder on how conscious intelligence is generated as a highly complex cognitive state in the human mind on the basis of biological and physiological structures" (Wang, 2009, p.1). This faculty, which was once believed to be limited to the human experience of reality, can now be present in AI systems. As the author highlights: “(...) the development of computers, robots, software agents, and autonomous systems indicates that intelligence may also be created or embodied by machines and man-made systems" (Wang, 2009, p.1). Wang (2009) explained that artificial intelligence is cognitively inspired and can be defined in terms of abstract intelligence, which he defines as a mechanism that transfuses information into specific behaviors and/or actions/activities. The human or system with abstract Intelligence possesses the faculty of transforming the information it acquires, ultimately creating knowledge, and performing actions that derive from it.

Another definition of AI is the one presented by Kok, Boers, Kosters, Putten \& Poel (2009). The authors state that, instead of trying to define Artificial Intelligence, one should focus on understanding the concept of intelligent systems. According to the authors, these usually fall under the following categories: "systems that think like humans; systems that act like humans; systems that think rationally; systems that act rationally" (Kok et al., 2009, p.2).

The concept of Hybrid Intelligence is described as a combination of human intelligence with Artificial Intelligence. Currently, AI algorithms excel at statistical tasks, such as the recognition of patterns, and perform them faster than humans. On the other hand, human intelligence is good at tasks that require adaptability, empathy, and common sense. Therefore, both types of intelligence are complementary to one another and can contribute to the uses of this technology in a way that highly benefits humanity (Dellermann, Ebel, Söllner \& Leimeister, 2019).

Furthermore, the field of Artificial Intelligence can be divided into a number of specific areas: machine learning (ML), natural language processing (NLP), expert systems, computer vision, speech, planning, and robotics (Kumar, 2018). Machine learning and computer vision are the two branches of AI that constitute crucial aspects of the functioning and understanding of the Style Transfer algorithm, which will be used in the empirical study of this dissertation (Jing, et al., 2019). In the next few paragraphs, definitions of these fields will be presented. 


\subsubsection{Different Types of Artificial Intelligence}

\subsubsection{Machine Learning}

In the book Understanding Machine Learning: From Theory to Algorithms, ShalevShwartz and Ben-David (2014) start by contextualizing the definition of machine learning through the concept of learning. The authors define learning as a movement where experience is transformed into knowledge or expertness. As such, machine learning is an area in which computer programs mimic the learning abilities of humans (Natarjan, 1991). According to Shavel-Shwartz \& Ben-David, machine learning relates to the recognition of determined patterns in data, "Machine Learning is an evolving branch of computational algorithms that are designed to emulate human intelligence by learning from the surrounding environment" (Naqa, Murphy \& Li, 2015, p.1). This branch of AI is used to create precise models based on the inputted data, such as predictive models, and is often applied in a number of systems, such as antispam software, and digital cameras (ShavelShwartz \& Ben-David, 2014).

The field of machine learning is subdivided into four main branches: supervised learning, unsupervised learning, reinforcement learning and deep learning (IBM, n.d.). Over the next few paragraphs, we'll briefly go over each one of them.

\section{Supervised Learning}

In supervised learning, we usually have a collection of data and a level of understanding of how it is classified. The goal of this type of learning is to learn to find patterns in that data through the use of training labels (IBM, n.d.). In the training process for supervised learning, an AI system might be provided, for instance, a series of images properly classified, train from them, and later be able to classify unlabeled images (Wilson, 2019).

\section{Unsupervised Learning}

Contrary to the previous one, unsupervised learning is characterized by learning about the presented data with no human intervention. While in supervised learning the data has been previously classified, in unsupervised learning the algorithm finds the patterns and clusters in the dataset and categorizes them. This type of learning is usually applied when there is an extensive volume of data that is unlabeled (IBM, n.d.).

\section{Reinforcement Learning}

Reinforcement learning works through a learning process of trial and error (IBM, n.d.). This could either be done through positive or negative reinforcement. In positive reinforcement learning the actions which help the algorithm achieve the desired goal will produce a "reward" which will increase the probability of them being repeated. With negative reinforcement learning, the actions that did not help in the achievement of the desired outcome will be avoided (Nelson, 2019).

\section{Deep Learning}

Deep learning is used to discern patterns in data that is unstructured. It is often applied in tasks involving visual recognition and computer vision. We can say that "deep learning complex neural networks are designed to emulate how the human brain works, so 
computers can be trained to deal with poorly defined abstractions and problems" (IBM, n.d.). This type of ML is particularly relevant for this work since it constitutes one of the underlying mechanisms that operate within Style Transfer (Gatys et al., 2016).

\subsubsection{Computer Vision}

A type of Artificial Intelligence that holds major importance for the making of this dissertation is computer vision. In the book Computer Vision: Algorithms and Applications (Szeliski, 2011), Szeliski starts defining the concept by sharing information about the human visual system. The author states that humans are able to recognize and distinguish the different objects that compose reality, they are also able to discern the objects from their background, by observing the shadows and light that interact with them. According to him, "perceptual psychologists have spent decades trying to understand how the visual system works and, even though they can devise optical illusions, to tease apart some of its principles, a complete solution to this puzzle remains elusive" (Szeliski, 2011, p.3).

Computer vision aims to collect information from images (Prince, 2012), as such, it attempts to denote algorithms with a similar perception to that of humans. To do this, programmers make use of fields that model physics (physics-based and probabilistic) in a way that aims to describe how objects behave in reality. These models analyze aspects such as light, shadow, movement, and other visual characteristics of the physical world (Szeliski, 2011).

The author also makes an important distinction between the fields computer vision and computer graphics, describing that while the first one aims to replicate everyday scenes by rendering specific scenarios and imitating the world in an "illusion of reality" (Szeliski, 2011, p.3), the second one attempts to do the opposite: "In computer vision, (...) we are trying to describe the world that we see in one or more images and to reconstruct its properties, such as shape, illumination, and color distributions" (Szeliski, 2011, p.3).

\section{Visual-imagery-based AI}

In relation to computer vision, the concept of imagery-based-AI proposed by Kunda (2018) is introduced. In the article "Visual Mental Imagery: A view from Artificial Intelligence", the author explores different questions regarding visual-imagery-based AI systems. In this work, the author categorizes visual-imagery-based AI systems, setting three principles that should be observable in this type of software and algorithms: "Criterion 1: Visualimagery-based representations must be 1) image-like, i.e., iconic, and 2) visual (...); Criterion 2: Visual-imagery-based representations must differ from concurrent perceptual inputs; (...) Criterion 3: Visual-imagery-based representations must play some functional role in performing intelligent tasks" (Kunda, 2018, pp.4-6). These constitute important concepts that help us further define and conceptualize this type of AI.

\subsection{Defining Creativity}

\subsubsection{Elements of Creativity}

Walia (2019) presents four defining aspects of creativity. The author analyzed the definitions of creativity from a series of different authors and found a common element to all - they characterized creativity as an act (Walia, 2019). Therefore, the first element established by this author describes creativity as an act. Defining the concept as the 
creation of something new. The second element presented by the author introduces creativity as production instead of a reproduction. The third element consists of the fact that creativity acknowledges a disequilibrium. Finally, the fourth element states that creativity is about sensitivity in perceiving a problem (Walia, 2019).

A definition of creativity is also presented by Sarkar and Chakrabarti (2015). Such as the previous one, the authors examined a wide group of definitions of the concept written by several professionals and researchers to define the fundamental aspects that characterize it:

Creativity is the generation of something that is both novel and valuable. On other terms, Creativity is an ability or process during which a person (or agent) generates 'something' that is 'novel' and 'valuable' (...). This 'something' can be a 'problem,' 'solution,' 'work,' 'artifact,' 'statement,' 'discovery,' 'thought,' 'idea,' or 'judgment' depending on the context. In the context of design, 'something' could be taken as 'problem,' 'solution,' 'product,' 'idea,' or 'evaluation (Sarkar \& Chakrabarti, 2015, p.16).

The authors also propose a table with a list of three important abilities that are present in creativity. The first one relates to the capacity of creating new ideas (Sarkar \& Chakrabarti, 2015). These ideas joined together, can give shape to what is referred to in the art's world as a concept. According to Hambeukers (2018), a valuable concept should stimulate the creation of new ideas and help create meaningful connections. Being able to properly communicate those ideas is crucial for the creation of multimedia products (Dolese, 2015). According to Osborne (1982) expressiveness is one of the attributes that might contribute to an effective communication of ideas and emotional states.

The second aspect Sarkar and Chakrabarti (2015) define as important in creativity is related to the ability of judging how novel the generated concepts/outputs are. According to Runco and Jaeger (2012), novelty and uniqueness are often characterized as integrating aspects of originality, a key element of creativity.

Finally, another crucial element is how one is able to perceive the usefulness of the result of the ideation process (Sarkar \& Chakrabarti, 2015). The previously presented definitions will be used throughout this dissertation for the discussion of the concepts and the understanding of the different aspects that will be analyzed.

\subsubsection{The Creative Agent}

For a deeper understanding of how creativity manifests, it is relevant to explore the characteristics that should be present in the creative individual. In the next paragraphs we will analyze the creative agent in the light of some important theories surrounding creativity. 
An author of relevance for the development of this work is Sternberg. In the article "The Triangular Theory of Creativity" (Sternberg, 2018), Sternberg analyzes the various factors that play a role in artistic creation. The development of this theory came from years of research. Initially, the author created other theories revolving around creativity, namely the investment theory of creativity and the propulsion theory of creative contributions (Sternberg, 2018). For context, the first one will be briefly described in the next few paragraphs.

The investment theory of creativity was developed with the participation of Todd Lubart. The key idea behind this theory is that creativity emerges as the result of a choice. The author argues that the biggest challenges to one's creativity come from the constraints an individual imposes on themselves: "The greatest obstacle to creativity, therefore, often is not exactly strictures from others, but rather the limitations one places on one's own thinking" (Sternberg, n.d.). Furthermore, the creative process can come from a variety of places and be the result of external inputs (Sternberg, 2018). In this theory, Sternberg (2018) underlines that creativity can be developed as a skill, detailing the numerous factors that greatly contribute to the enhancement of the ability.

Firstly, it is important to allow people to create new ways of perceiving and approaching problems. Secondly, one should allow themselves to moderately experiment within the realm of chaos. Thirdly, it is incredibly relevant to pitch and consider new ideas, regardless of the likelihood of acceptance from the other people's part. The fourth characteristic expressed as relevant consists in being persistent in the emergence of possible blockages or impediments. Finally, an individual should be able to self-analyze and understand if their own prejudgment is standing in the way of their abilities to explore creativity (Sternberg, 2018).

The author explains that the creative process is greatly impacted by a variety of different aspects, such as "(a) abilities, (b) knowledge, (c) styles of thinking, (d) personality attributes, (e) motivation, and especially intrinsic motivation, and (f) environment" (Sternberg, n.d.). He states that a subject could have the inherent creative ability, but due to environmental constraints be limited in the expression of that capability. Moreover, “(...) individuals need be not just defiant; they also must have the analytical skills to assess the quality of their ideas, the practical skills to persuade others of the value of their ideas, the passion to pursue their creative ideas to their ends, and the resilience to persist in the face of opposition" (Sternberg, 2018, p.52). This theory served as an important foundation for the development of a theory that is relevant in the context of this dissertation, the triangular theory of creativity (Sternberg, 2018).

In the triangular theory of creativity, Sternberg (2018) discusses the important underlying aspects for the development of creativity, empathizing how one's challenging and reconfiguring its model of reality might highly impact their creative endeavors: “(...) optimal levels of creativity result not just from defying the crowd - that is, other people with more conventional conscious beliefs - but also, from defying oneself and one's own beliefs as well as defying the usually unrecognized and perhaps unconscious field-based presuppositions (the Zeitgeist) upon which one's own and others' beliefs are embedded" (Sternberg, 2018, p.4).

In the triangular theory of creativity, Sternberg (2018) distinguishes three types of defiance. The first one consists of the defiance of the crowd. The author argues that some 
of the most brilliant minds have struggled through this aspect. That is "(...) difficult because creative people, perhaps even more than some uncreative ones, want to be appreciated for their work" (Sternberg, 2018, p.4). While it might initially be difficult for the individual, Sternberg defends, that this aspect is crucial for the development of this faculty and that it will pay off in the long run in terms of his/her creative endeavors (Sternberg, 2018).

The second aspect consists of defying oneself. The author states that this one might be even more challenging than the previous one and that it consists of a facet that had been initially discussed in the investment theory of creativity, but not through the right lenses. Sternberg highlights that the reason why an individual may have difficulty confronting themselves is due to the fact that they have settled views of themselves and the world, projecting any obstacles preventing them from reaching their full potential on other people and the surrounding environment. Moreover, people looking for being creative "(...) not just once, but repeatedly throughout a career" (Sternberg, 2018, p.6), need to defy their own previous ideas.

Finally, the third important aspect subsists in the defiance of the Zeitgeist. This aspect refers to the cultural environment of a particular era and often connects with the "(...) set of beliefs we or others (the crowd) are aware of having but rather the set of presuppositions we often do not even consciously know we have" (Sternberg, 2018, p.6). The author argues that often, new research, perspectives, and theories on scientific endeavors emerge in a determined cultural context that influences their appearance and development (Sternberg, 2018).

\subsubsection{The Creative Process}

In order to gain a deeper understanding of the creative product, the object that is evaluated in this dissertation, it is crucial to analyze the creative process. In the next paragraphs the model of components by Amabile (1996) and the definition of the creative process by Taylor (2017), will be presented.

The relevance of Amabile's work for this dissertation relates to how her models contribute to the understanding of the underlying factors that propel and influence the creative process and its exploration. According to this author, creativity merges together the following mechanisms: an intrinsic motivation, knowledge on the topic which corresponds to expertise and other processes that relate to creativity (which are described in the form of abilities) (Amabile, 1996). According to Taylor (2017), innovation is an important aspect of the creative process. The author describes this process as the convergence of creativity and innovation (which are tightly linked) with the goal of originating something valuable

The key concept around which this theory revolves is Intrinsic Motivation. This coincides with the motivation that resides inside of the individual, which differs from externally affected motivation. According to the author, it is this type of motivation that propels artistic creation. External motivation, on the other hand, refers to an impulse that comes from the outer world, such as an unexpected evaluation or reward. Amabile (1996) defends that external motivation, in case of it not being controlling, will not necessarily block or negatively affect creative expression, but it also will not act as an enhancer towards it. 


\subsubsection{The Creative Product}

Another important aspect to consider for this dissertation on the evaluation of the creative artifacts is the definition of the creative product. Amabile (1982) characterizes this term, and relates it to a definition of creativity:

A product or response is creative to the extent that appropriate observers independently agree it is creative. Appropriate observers are those familiar with the domain in which the product was created or the response articulated. Thus, creativity can be regarded as the quality of products or responses judged to be creative by appropriate observers, and it can also be regarded as the process by which something so judged is produced (Amabile, 1982, p.1001).

The author affirms that analysis and evaluation of creativity should be done through the observation of a product, even when the focus is on the process since the first corresponds to the materialized result of the second. Therefore, individual characteristics that indicate creativity should always be analyzed together with the quality of the subject's work (Amabile, 1982).

Amabile shares that, even though creativity might at times be hard to perceive when referring to specific characteristics that are inherent to the individuals, it is something that can easily be spotted when observed materialized through a product, making this an ideal approach for empirical research. The author states that individuals acquainted with those products (experts) are in agreement with each other to a certain level in regard to their observations on creativity expressed through them, so "(...) if appropriate judges independently agree that a given product is highly creative, then it can and must be accepted as such" (Amabile, 1982, p.1002).

The previously introduced definition complements the characterization of the elements of creativity presented by Sarkar and Chakrabarti (2015), and Walia (2019). They proposed a way in which those elements can more easily be observed and analyzed, through the evaluation of artifacts. In the present research, the previously defined characteristics of creativity, presented by Sarkar and Chakrabarti (2015) and Amabile (1982), will be analyzed in the context of the outputted videos transformed through the use of Style Transfer.

\subsubsection{Computational Creativity}

In the context of this dissertation, another aspect that is greatly relevant to present is the concept of computational creativity. This consists of a field that studies the development of software that generates creative work. These creativity abilities can be applied in a series of areas that range from mathematics to fine arts (like music and painting) and even writing (Colton, López de Mántaras, \& Stock, 2009). 
According to Park (2019), humans have longed for the creation of something that transcends our species. This author emphasizes that "as our minds can run beyond the boundaries created by our body limitations, we would like to infuse our creativity into AI that might evolve from its original state. Similar to what Prometheus did, humans are attempting to share their legacy with another existence" (Park, 2019, p.102).

Computational creativity has become a very pertinent area over the past few decades, as a lot of issues concerning algorithmic art creation have been brought to light (López de Mántaras, 2013). One of the debated topics is surrounding the Turing test, which aims to determine if software could pass as a human in a number of areas (Oppy \& Dowe, 2019). In regard to artistic creation, the Turing test aims to verify if the works produced by AI can come close in terms of artistic value to those built by humans, that is if the subjects are able to discern the works of a machine from the ones generated by people (López de Mántaras, 2013)

According to López de Mántaras (2013), the use of machines and algorithms for creative production has been increasing in multiple artistic areas. The author states that "(...) the computer is already a canvas, a brush, a musical instrument, and so on" (López de Mántaras, 2013, p.1). The author affirms that it is important to set aspiring goals in terms of computational creativity. Mántaras thinks of creativity as a result of reasoning processes that involve a series of other underlying cognitive functions, some of which are: memory, analogy, and learning processes. The author explains that these can be simulated through the use of software. Even though we do not hold yet a full understanding of some of the underlying functions of these abilities that contribute to artistic creation, over the last few years, we've been perfecting our AI tool's capabilities of mimicking them (López de Mántaras, 2013).

After interviewing several experts, Ornes (2019) underlined that creative ability was previously believed to be a trait unique to humans. Over the past few years, the question of if an algorithm can, independently, create artwork that would be indiscernible from work made by humans has been raised. In case the answer is yes, what would be the implications? Would this mean the software and algorithms can be seen as creative, or that they are simply imitating humans in their creations (Ornes, 2019)? Late developments on artificial intelligence systems challenge the notion of humanity holding exclusivity in art creation. There has been a movement in the computer science community that aims to generate algorithms with creative abilities. This means that the output of those algorithms contains the core elements that would cause it to be considered creative if it was generated by humans (Ornes, 2019).

Some authors, such as Ornes (2019), stress the idea that the usefulness of these systems exists merely as a tool. In "Driving The Creative Machine", Cohen (2010) affirms that machines and algorithms will always be dependent on humans: "(...) the best-designed set of mechanisms will not accomplish much without an engine to drive them, and that the engine is provided for a creative human agent" (Cohen, 2010, p.16).

Others, such as Park (2019), see the possibility of viewing AI software as creative agents as something that can soon become a reality: "AI (...) that can self-reflect and modify what it does generates various images from the learning system just like an art majored student does" (Park, 2019, p.119). Moura, an artist that creates art through the use of AI and Robotics states that "Whether a work of art is made directly by a human artist or is the 
product of any other type of process is nowadays of no relevance" (Moura, 2018, p.3). The author affirms that both types of artistic creations should be accepted, as there are various examples of alternative creative processes throughout the history of art that were widely accepted, naming the most important factor to consider something as art or not as the level of expansion a certain technique brings to the artistic field (Moura, 2018).

Some individuals resist the acceptance of AI as potentially creative, like King, the founder of an AI company that works with creativity. In an interview conducted by Bonini and Donoughue (2017), King states that AI's abilities are limited to what it has been trained to do, unlike human abilities which usually transcend that. McCormack, an artist and computer scientist, in an interview with Bonini and Donoughue (2017), affirmed that the essence of art lies within artistic communication, as such, when having a computer perform such a task, it would be difficult to classify the work as truly artistic.

Finally, others, like Audry and Ippolito (2019) state that whether AI can be truly creative or not is not the right question and that we should instead ask what changes this brings to artistic production, more specifically, "what roles does machine-made art leave for artists - imagined or real, flesh or silicon - and the viewers who imagine them" (Audry and Ippolito, 2019, p.8). Regardless of the answer, the possibility of measuring creativity seems to be relevant and can contribute to a better understanding of the relationship between creative art and AI systems.

\subsubsection{Measuring Creativity}

Considering in this study we aim to understand if the use of an AI algorithm contributes to changes in the perceived creativity of the videos, it is extremely important to understand how those changes can be measured. In the next few paragraphs, we will briefly present the different categories of tests that measure creativity and specify the type of test that was used for this research.

Sarkar \& Chakrabarti (2015) analyzed a series of 74 creativity tests and divided them into five different categories, according to the different approaches. The first category is "Ability-based tests". This refers to tests that seek to measure the strength of the subject's creative capabilities. The second category, "Character-based tests", works through the identification of characteristics of an individual's character that are believed to be fundamental aspects that contribute to the creative ability. Thirdly, there's "Determination of past creative activities". These tests aim to measure creativity through the amount of creative tasks performed in the past (Sarkar \& Chakrabarti, 2015).

The fourth set of tests presented by the authors are "Outcome-based tests", which work through the analysis of creative outcomes. These evaluate the creative product instead of focusing on the individual. According to these authors "based on the characteristics of the outcomes created by the individuals, these tests decide on the occurrence of creativity" (Sarkar \& Chakrabarti, 2015, p.19). Some of the tests present in this group include the Creative Product Semantic Scale (CPSS), the Consensual Assessment Technique (CAT), and the Student Product Assessment Form (Sarkar \& Chakrabarti, 2015). Since this dissertation focuses on the product, the "Outcome-based tests" were the type of creativity tests that were employed. 
Finally, the last group of tests classified refers to "Environment suitability tests". In this type it is considered that a specific environment is a factor that contributes to the development of creative talent and creativity enhancement. As such, these tests seek to identify the creative context of the individual (Sarkar \& Chakrabarti, 2015).

\subsection{Artificial Intelligence Software and Algorithms for Creative Use}

Over the past decades, numerous algorithms have been generated to be used in the creation of artistic products, such as the one employed by Aaron, a robot that runs on an algorithm programmed by Cohen, a visual artist. The created algorithm has been perfected throughout the years and is used by the artist in the creation of his paintings (Cohen, 2010). Another example is the (CAN) Creative Adversarial Networks system, proposed by Elgammal, Liu, Elhoseiny and Mazzone (2017), which outputs novel art pieces inspired by a series of paintings (Elgammal et al., 2017), and Sony's Flow Machines technologies, an AI system used for music creation (Avdeeff, 2019). In the next few paragraphs, these and multiple other systems will be presented and described.

\subsubsection{Style Transfer in Real-Time}

As mentioned above, Style Transfer is the Artificial Intelligence algorithm that will be used in the making of this dissertation. In this system, the AI system captures different characteristics of the input image, separating it from the objects in the frame and applying it to other images to create new stylized content (Gatys et al., 2016). The Style Transfer algorithm used for the empirical study is the one composed by Jin (2018). The code aimed to improve on earlier works. This algorithm analyzes the characteristics of a style of an artistic image (inputted by the user) and learns to distinguish those from the elements in the frame to be able to apply the same style to other visual content, both images and videos (Jin, 2018). The programming language in which it has been made is Python and it makes use of the following libraries:

TensorFlow, an open-source framework that was generated by the Google Brain Team, which contributes to the building of models for machine learning. It contains a series of models and algorithms for neural networks (Yegulalp, 2019).

NumPy (Numerical Python), a package for Python, one of the main libraries used for scientific computations, which is often applied for processing that involves arrays. It is a relevant tool in works that include areas such as machine learning and statistics (DeepAI, n.d.-a).

SciPy is a Python library. It is often applied in the computation of scientific/mathematical tasks. This tool is built inside of Numpy. "Both NumPy and SciPy are Python libraries used for mathematical and numerical analysis. NumPy contains array data and basic operations such as sorting, indexing, etc, whereas SciPy consists of all the numerical code" (Urooj, 2019).

Finally, we have MoviePy, which is an important tool for basic video editing. It allows transformations such as cuts and title insertions. MoviePy may also be used for the generation of advanced effects and the processing of videos (Zulko, 2017).

This algorithm was chosen for two reasons. first, because it will allow the study of the AI algorithm's impact on audiovisual pieces. The second reason is that, even though AI 
algorithms have been used in empirical studies related to creativity, Style Transfer remains unexplored. In the next few paragraphs, the algorithms that inspired the creation of that one used in this study will be presented, as well as other cases of Artificial Intelligence software and algorithms used in artistic production.

\subsubsection{Style Transfer for Images through the use of Convolutional Neural Networks}

The first algorithm that will be introduced is the one presented by Gatys et al. (2016) in "Image Style Transfer Using Convolutional Neural Networks". In the creation of this system, the authors resorted to convolutional neural networks (CNNs) that work by analyzing an input image and learning to distinguish between the various elements that compose it. This neural network system attempts to mimic human vision (Gatys et al., 2016).

The authors propose an algorithm that can specifically pinpoint and distinguish between shapes in a way that allows it to discriminate between the object itself and the style of the image. The algorithm can then be used to output new images, by applying the style of a particular painting in a photograph, resulting in it resembling an artwork by a determined artist (Gatys et al., 2016).

CNNs have three main types of layers that should be presented for a better understanding of the underlying functioning of the system. The first layer is the convolution layer, known as The Kernel. There is usually more than one in a network. The first convolutional layer is often used to extract more low-level features (like color and edges), as more layers are added, the high-level features are then generated from the combination of the low-level features, which contribute to the understanding of the images (Saha, 2018). In regard to the use of higher vs lower layers in the network, the authors share the different ways in which this impacted the visual results of the Style Transfer.

When matching the content on a lower layer of the network, the algorithm matches much of the detailed pixel information in the photograph and the generated image appears as if the texture of the artwork is merely blended over the photograph (...). In contrast, when matching the content features on a higher layer of the network, detailed pixel information of the photograph is not as strongly constraint and the texture of the artwork and the content of the photograph are properly merged (Gayts et al., 2016, p. 2420).

The second layer that will be presented is the pooling layer. This one works by decreasing the spatial size of the convolved feature (see figure 1). This has two benefits, first of all, it reduces the amount of power that is necessary to process the information, secondly, it helps separate the main features (Saha, 2018). 


\begin{tabular}{|c|c|c|c|}
\hline 0.1 & 0.5 & 1.2 & -0.7 \\
\hline 0.8 & -0.2 & -0.5 & 0.3 \\
\hline 0.4 & 0.9 & -0.1 & -0.2 \\
\hline-0.6 & 0.1 & 0.5 & 0.3 \\
\hline
\end{tabular}

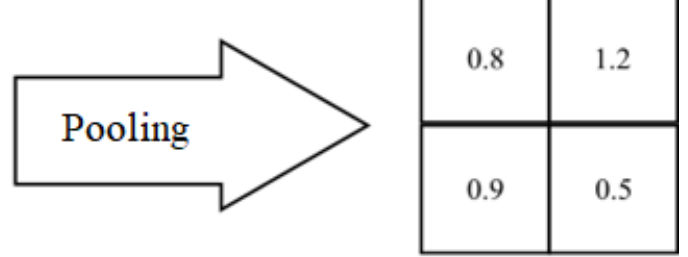

Figure 1. Pooling.

Finally, there's the fully connected layer, in which every node connects to all the other nodes in the network (Data Science Glossary, 2019). This helps the network learn about the non-linear combinations between the high-level features (Saha, 2018).

The authors propose an explanation as to why this network happens to perform so well in distinguishing the style from the other elements of the image, stating that when learning about how to discriminate the various objects, the network needs to be invariant in regard to the visual transformations of the images that still maintain the identity of the object. Furthermore, "representations that factorize the variation in the content of an image and the variation in its appearance would be extremely practical for this task" (Gatys et al., 2016, p. 2421). This work was one of the sources of inspiration for the creation of the Style Transfer algorithm that is used in the empirical study of this dissertation.

\subsubsection{An optimized version of Style Transfer through the use of Perpetual Losses}

Another Style Transfer algorithm that contributed to the building of the one used in the present study is the one described in "Perceptual Losses for Real-Time Style Transfer and Super Resolution" by Johnson, Alahi, and Fei-Fei (2016). In their article, the authors made an optimization of the network proposed by Gatys et al. (2016), in which they combined per-pixel loss (between the input and output images), with perceptual loss (Johnson et al., 2016). Per-pixel loss is a function that aims to help the network produce visual results faster by capturing the differences between the images on a pixel level (Johnson et al., 2016). This is different from perceptual loss, which instead of analyzing the differences between pixels, processes the disparities between the high and low-level features (DeepAI, n.d.-b).

The authors state that compared to the method presented by Gatys et al. (2016), this system shows improved performance, while maintaining the same level of quality. They highlight that it is three times faster than the original algorithm (Johnson et al., 2016).

\subsubsection{Optimization of Style Transfer through the use of Instance Normalization}

Another algorithm that also aimed at speeding up the stylization process, and that consists of an important reference that inspired the development of the Style Transfer tool used in this study, is the one presented by Ulyanov, Vedaldi and Lempitsky (2017) in the article "Instance Normalization: The Missing Ingredient for Fast Stylization". In the context of the lack of efficiency in the time taken to process each individual frame of the method 
proposed by Gatys et al. (2016), two methods that aim to enhance the performance were introduced, proposed by Ulyanov, Lebedev, Vedaldi, and Lempistky (2016) and by Johnson et al., (2016). Both systems make use of learning equivalent feed-forward generator networks, which, contrary to the first method, can create an image from a single pass (Ulyanov et al., 2017).

Even though these methods increase the speed, one aspect gets affected: the quality of the results. This paper presents an alteration to the code by Ulyanov et al. (2016) that can increase the quality of the output and maintain the processing speed (Ulyanov et al., 2017). To explain how this method was achieved, we will briefly go over some concepts that are important for the understanding of the functioning of the algorithm.

In machine learning, the training of the data is done through several passes/cycles. When the training of the neural network is being processed, and the amount of data that is used is too big for it to be processed all at once, the data is compartmentalized into smaller "pieces", to be fed into the algorithm one at a time. These are called batches (Sharma, 2017). Batch normalization can then be performed. This method standardizes the inputs to a determined layer (for each batch) and normalizes the learning process, which causes a scaling down in the amount of training epochs (Brownlee, 2019). Epochs are cycles of training of the entire dataset required in the training of the network (DeepAI, n.d.-c). In this method batch normalization is replaced by instance normalization, which is a technique that performs similarly in terms of speed, while increasing the quality of the computed output (Ulyanov et al., 2017).

\subsubsection{Style Transfer for Moving Pictures}

In the context of this dissertation, an algorithm of Style Transfer that holds special importance is the one developed for videos and spherical images by Ruder, Dosovitskiy, and Brox (2016). This differs from the previously presented algorithms because it focuses specifically on Style Transfer for audiovisual content (Ruder et al., 2016). These authors introduce two algorithmic proposals that assign the style of a determined image to a video. The approaches were created through the transformation of the Style Transfer algorithm built by Gatys et al. (2016). In this system, the authors propose techniques that aim to help in the generation of video in a coherent and cohesive manner and close to real-time.

Ruder et al. (2016), explain that the stylization of frames independently from one another leads to problems in the output video, producing visual disparities between the frames, which affects the coherence of the final output. In order to regulate this, temporal constraint was proposed. In their own words: “(...) instead of penalizing the deviations from the previous frame, we penalize deviation along the point trajectories" (Ruder et al., 2016).

The authors explain that when an area of the image becomes occluded in a set of frames, and after that is disoccluded again, it is likely going to be assigned a different visual appearance each time and, therefore, compromising the feeling of continuity between the several frames. The approaches presented in this article aim to solve this, through the use of long term estimates (Ruder et al., 2016). This is a technique that is often applied in the processing of audiovisual content, for tasks like the compression of videos (Smolic, Sikora, \& Ohm, 1999). 
Another aspect that was important to take into consideration was the appearance of artifacts in the corners of the images. In order to solve this, a multi-pass algorithm was generated, which works by exchanging temporal directions during the treatment of the video, both forward and backward. This technique produces high-quality outputs, however, as a drawback, its computation takes longer (Ruder et al., 2016). The solution presented in this paper allows the application of Style Transfer on videos while maintaining temporal consistency and visual coherence, which, consequently, generates an output that is more visually cohesive and aesthetically appealing.

\subsubsection{Creating Art Pieces Through the Use of Creative Adversarial Networks}

Previously, Style Transfer algorithms that have been produced for the stylization of videos were presented. In the next few paragraphs, a system that generates artworks by learning from inputted art pieces will be introduced. This system was created by Elgammal et al. (2017).

The underlying mechanism functions as follows: first, a series of images and artworks are presented to the system, which "learns" from them the various characteristics of the creative products. This is achieved through the use of Generative Adversarial Networks (GAN). The system was constructed in a way that propels it to create pieces that deviate from the original artworks that were used as input, just as a student in a classroom would be encouraged to look at art pieces from previous artists but ultimately motivated to create something unique and different (Elgammal et al., 2017). The authors aimed at the creation of a system that could produce artworks that caused arousal, through novelty, but without going to an extreme on that level, as according to the researchers, too much arousal could cause aversion. According to them, "this criterion is common in many computationally creative systems, however, it is not easy to find a way to achieve that goal given the infinite possibilities in the creative space" (Elgammal et al., 2017, p.1).

The procedure goes as follows: a Creative Adversarial Network (CAN) was developed, this network worked within two opposite forces with the goal of being able to create works that inspire novelty, however, without deviating too much from the previous pieces. Furthermore, "according to the Wundt curve (...), the generated work should increase the stylistic ambiguity" (Elgammal, et al., 2017, p.6).

These networks function in accordance with two different systems, one that consists of a discriminator and another one that is a generator. The art styles that are fed into the first one will be classified according to various art movements, with the goal of teaching it about the visual characteristics of each one, so it can distinguish between them. The second one (the generator) does not contact with the collection of artworks, as such, it creates works randomly. Then the first mechanism (discriminator) works in accordance with this one to classify the various works, dividing them into two groups with the labels of "Art" or "Not Art". The authors stated that this helps it "deceive" the discriminator, "(...) this signal enables the generator to change its weights to generate images that more frequently will deceive the discriminator (...)" (Elgammal, et al., 2017, p.6). The generator receives feedback on how easily the discriminator can classify the art pieces into one of the established styles, and in CANS “(...) the creative generator will try to generate art that confuses the discriminator (see Figure 2). On one hand it tries to fool the discriminator to think it is "art," and on the other hand it tries to confuse the discriminator about the style of the work generated" (Elgammal, et al., 2017, p.6). 


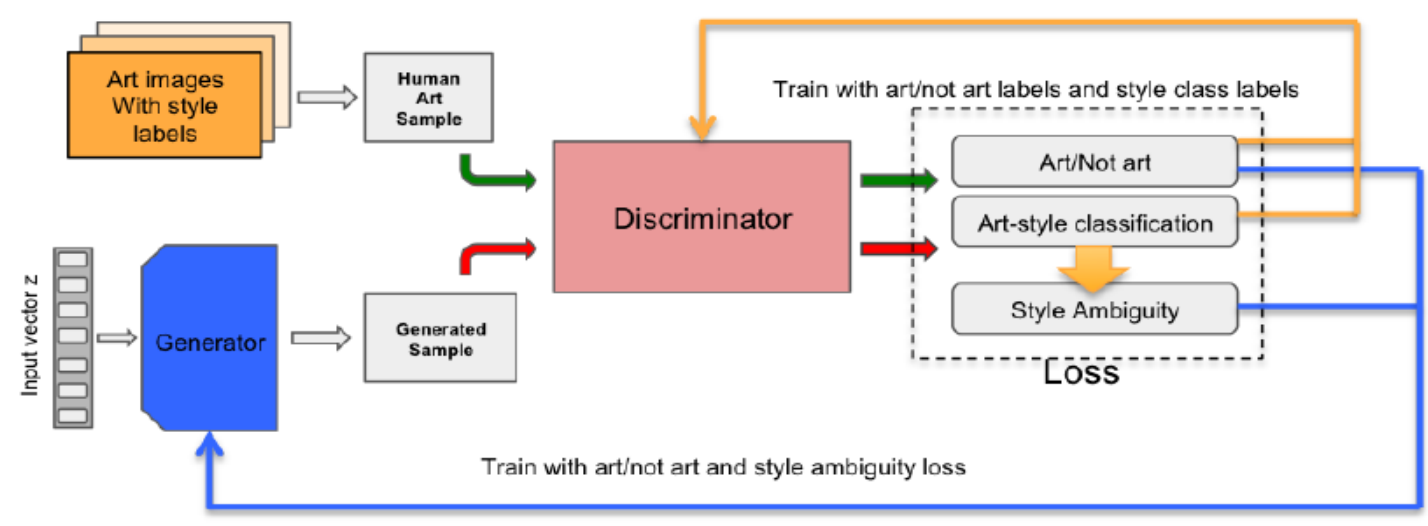

Figure 2. CAN System.

This system is of particular importance, due to the innovative algorithmic approach and the creative nature of the outputs. Later, a study done through the use of this algorithm will be presented. This study aimed at comparing the creative output of the algorithm with art pieces generated by humans.

\subsubsection{Artificial Intelligence in The Creation of Paintings}

In the previous pages, some important algorithms that were sources of inspiration for the Style Transfer used in this research were described and a CAN system for the creation of artworks was introduced. In the next few paragraphs, some cases of the use of Artificial Intelligence algorithms for the conception of paintings will be briefly presented.

One of the most relevant cases of the use of AI in painting is the one of Aaron, a robotic AI system that was generated by the artist Cohen, an avant-garde painter, engineer, and computer artist. Aaron was capable of drawing and painting and it played an important role in the production of Cohen's paintings, being his artistic partner in numerous of his creations (Garcia, 2016). The author worked in partnership with the robot for more than 40 years. During this time he explored different facets of the machine, taught it to perform a wide number tasks and continuously perfected its structure and algorithm (Cohen, 2010).

Another case of the use of robotics and AI in painting is the one of Moura. The author created a series of art robots that he uses to generate art pieces (Moura, 2018). The mechanism works as follows: a microchip and a series of sensors allow them to move without going against obstacles, to recognize colors, and draw through the use of a pen.

They move in a haphazard way inside an arena (...) but with each sensing the colour over which it is then passing and reacting by either raising or lowering its pen when a certain threshold is sensed, that is, when a certain amount of colour is present. This reaction to the marks left by other robots is thus an indirect form of communication (Moura, 2018, p.1). 
In regard to the relevance of the use of art-making machines for creative production the author states that knowledge evolves through experimentation and random exploration, as such, those aspects represent crucial steps for discovering the potential of a determined field. Moura creates an analogy between the evolution of species and the development of art, stating that it is important to produce more artworks through the use of this technology, in order to bring to light the potential of the use of AI and robotics for creative production (Moura, 2018).

\subsubsection{Other Relevant Artificial Intelligence Algorithms for Creative Production}

In the next few paragraphs, other AI technologies that have been used in a variety of areas for creative production will be briefly presented.

An important AI system is Deep Dream, created by Google. This system makes use of neural networks to detect determined patterns in the inputted images, generating a stylistic outcome that Google refers to as inceptionism (Mordvintsev, Olah, \& Tyka, 2015). The neural networks are trained by observing millions of images and learning to capture the high-level features present in them. When the training is finished, the networks are able to "identify" those features in other images (Mordvintsev et al., 2015).

Akten, as quoted by Rayner (2016) states "It might look like Deep Dream is generating say, sparrow's faces in clouds, but what it is actually doing is generating patterned noise, which our brains try to find meaning in". Although this system has not been specifically generated for creative and artistic production, a series of artists, such as Akten, have successfully appropriated this as a tool for their creative endeavors (Rayner, 2016).

The creative uses of Artificial Intelligence are not exclusive to the field of arts. An example of an algorithm that makes use of this technology for other creative applications is the Artificial Intelligence system for design ideation by Chen et al. (2019). This works through the use of Artificial Intelligence and data mining (a process that seeks to capture anomalies, patterns, and relationships in order to make predictions) (SAS, n.d.). A generative adversarial network outputs images blending different concepts, which then contribute to the generation of new visual ideas (Chen et al., 2019). The authors state that this model's application is not restricted to visual ideation and that it could also be used in other areas like advertising and commercial ideation (Chen et al., 2019).

Up until this point, visual applications of Artificial Intelligence have been presented. However, AI's uses are not restricted to imagery-based tasks. A series of algorithms have also been created for artistic use in several different areas. We present as an example AI Duet. This consists of a music-piano-bot software that was generated by Google, which has been trained using a dataset with multiple musical examples. The system works by analyzing the inputted notes and musically responding to them, complementing the user's creation (Statt, 2017). The idea is "to get everyday users to play around with these experiments to help inform how they're built and how they can be improved, as well as to help independent developers and musicians create new experiences using powerful AI learning software" (Statt, 2017).

Another non-imagery-based AI is Shelley. Shelley: Human-AI Collaborated Horror Stories is a project created by the MIT media lab, in which a deep learning algorithm learns how to write horror stories by reading texts from the subreddit "nosleep". After the training, the 
subjects can participate in the writing of these stories with Shelley, by commenting on the beginning of a story in the twitter account created for the character, which will give continuity to their narrative (Mit media lab, n.d.).

\subsection{Empirical Research}

\subsubsection{AI vs Human-Generated Art}

Since AI is a fairly recent area of study, there have not been conducted a large number of studies that relate the use of AI systems with creative productions, after an extensive search, the following were highlighted as the most important sources of inspiration for the conception of this research.

Firstly the study by Elgammal et al. (2017) concerning Generative Adversarial Networks. In the research, GANs were used as a way of bringing artistic works to life. The process goes as follows: first, the artist selects numerous artworks, then it feeds those to an algorithm. The algorithm functions in between two opposing forces, one that makes it create new pieces by attempting to mimic those images that were used as input, and another one that pushes it into creating something different (Mazzone \& Elgammal, 2019).

After the works were created, the researchers performed an assessment: a group of individuals were asked to analyze the pieces in terms of creativity, with the aim of understanding whether the subjects could discriminate works made by humans from the ones generated by AI. The authors also made use of qualitative analysis in order to better understand which subjective characteristics the subjects projected unto the artworks (Elgammal et al., 2017). For this part, there were selected two collections of works created by human artists and four groups of works created by the system (Elgammal et al., 2017). The following research was conducted through a series of experiments, which will be described and analyzed through the next paragraphs.

The first experiment carried out by the authors aimed to understand if the individuals were able to distinguish art created through this system from other types of art. After being shown the images, the subjects were asked about whether they thought the work was created by an artist or generated by a computer and to assess the image on a Likert scale (Elgammal et al., 2017).

The following experiment aimed to understand if the images generated by CAN could be classified as art. In this one, a group of images created through the use of CAN were compared with artistic images from the Art Expressionist and the Art Basel collections (Elgammal et al., 2017). The questions asked revolved around the individual's subjective impression of each image if they considered it appealing, novel, surprising, ambiguous, complex, and finally, if they thought it had been created by a human or by a computer. Once again, the researchers resorted to a Likert scale to measure this aspect. In the next part of the experiment, questions related to the qualitative nature of the works were presented. In this phase, the participants were asked if they saw intentionality and structure in the paintings. They were also inquired about whether they considered the piece communicated with them and inspired them. Finally, for the last experiment, the objective was to understand if implementing the "style ambiguity loss to the CAN model" (Elgammal et al., 2017, p.18 would have an impact on the public's perception of the pieces. The experiment went as follows: “(...) in order to evaluate novelty we used a pool 
of art history students as sophisticated art-educated subjects who can judge the novelty and aesthetics better than general MTurk subjects" (Elgammal et al., 2017, p.18). The images were presented to the individuals organized in groups of two (side by side).

Two questions were presented in the last phase. The first one related to the novelty of the image. In this one, the individuals had to state which image they considered more novel. The second one was in regard to the aesthetics of the pieces. The participants had to pick the image that they considered the most aesthetically appealing from the presented collection. The study concluded that frequently, the human subjects could not discriminate AI art from human-created art (approximately $75 \%$ percent of the time). Besides this, subjects described the works with the terms "(...) "intentional", "having visual structure", "inspiring", and "communicative" at the same levels as the human-created art" (Mazzone \& Elgammal, 2019, p.5).

\subsubsection{The Perception of the Identity of The Creative Agent}

Another relevant study is the one conducted by Hong and Curran (2019). In their research, the authors explored if the perception of the creativity of several art pieces was impacted by what was perceived to be the identity of the author (Artificial Intelligence system or Human). This study was relevant, not only because it deals with a similar thematic (the perception of art pieces) but also because it is built on an extremely concise and meticulous method. First, the individuals were randomly selected. Those who suspected what the study was about were excluded from the study and the other ones remained. Next, the participants were shown a collection of art pieces by AI and human artists and asked to rate them on a variety of aspects on a Likert scale from 1 to 5 often applied by professionals for the measuring of creativity in artworks. The scale that was given to the participants makes use of the following aspects to measure the creativity of the artworks: "(...) originality, degree of improvement or growth, composition, development of personal style, the degree of expression, experimentation and risk-taking, aesthetic value, and successful communication of ideas (...)" (Hong \& Curran, 2019, p.9).

The results were later measured through the use of a t-test, and the values of the several groups were compared, Contrary to the previous study presented by Elgammal et al. (2017) this study, concludes that (1) there are still differences between the ratings of the artworks generated by Artificial Intelligence and the ones created by humans, as such, it appears that the outputs of the algorithm do not show the same level of creativity as human-made artworks and that (2), the perceived identity of the artist does not affect the rating of the artworks (Hong \& Curran, 2019).

This study concluded criticizing the one conducted by Elgammal et al., (2017), stating that its method lacked accuracy, and presenting results that lead to a different conclusion, that AI-generated artworks have not yet reached the level of perceived creativity of humancreated art pieces. The authors state that, first of all, asking questions directly about the nature of the artworks might produce a bias in the responses. The second aspect that was criticized was the small number of participants (20), which the authors state is not enough to generalize the obtained results (Hong \& Curran, 2019). Future studies should address those two issues raised by Hong and Curran. 


\subsubsection{Artificial Intelligence Software for Ideation}

Finally, another study that was made, regarding the use of AI for creative production, was the one conducted by Chen et al. (2019). In this study, the authors proposed an AI-based, data-driven approach to ideation for design, through the use of generative adversarial networks. It was performed a design case study to see if this system would contribute to the ideation process. In this study, both experts and non-experts in design participated. The subjects were distributed through two groups, a treatment group, and a control group. The participants in the treatment group made use of the created system. The participants in the control group did not have access to this tool, however, they could search in Google to help with the ideation process. The sample consisted of engineering students, but only two subjects of each group had studied design engineering. The study concluded that the approach can help create multiple different associations between concepts from different categories, and assist in the process of generation of new ideas in a fast and easy manner, leading to a higher quantity and novelty of the generated products and concepts (Chen et al.,2019). This study presents AI as a tool for creative Ideation. However, it does not test the level of creativity of the concepts generated.

Considering all the studies here summarized, some ideas come up:

a. Although AI seems to be able to produce some of the same attributes of creativity as humans (as perceived by people), it is unable to produce artworks to an equal level of creativity (Elgammal et al., 2017; Hong \& Curran, 2019). Therefore, new studies on the creativity of the generated outputs are useful for clarifying this issue;

b. The artworks generated in the described studies were paintings and drawings. Therefore, it is important to verify to what extent other artistic forms (like video) can benefit from the use of Artificial Intelligence systems. New studies addressing this issue seem to be relevant;

c. Following the best practices of using experts and non-experts, future studies, where possible, should use participants from the two groups;

d. Following also the best practices concerning the type of data collected, future studies should measure, at the same time, qualitative and quantitative aspects of creativity;

e. So far, to our knowledge, the research undertaken on creativity and AI has used GAN and CAN systems. None of the studies have employed Style Transfer algorithms. This is relevant since that algorithm allows us to compare the same video creations transformed with an Artificial Intelligence (AI) algorithm and without the use of Artificial Intelligence (No-AI), making more reliable and accurate comparisons between AI and No-AI.

Those challenges are considered in the empirical research undertaken within the scope of the present dissertation which is described below. 


\section{Method}

\subsection{Experimental Study}

Aiming at verifying to what extent an AI algorithm improves the creativity of videos, an empirical research was carried out. The study here reported has an experimental research design. Twelve videos were used, six for verifying the similarity between experimental and control groups regarding the evaluation of the creativity of the videos, and the other six for comparing the evaluation of AI and no-AI versions of the videos. The Style Transfer algorithm was used to transform the videos. Both quantitative and qualitative data were collected as the two types of data complement each other, and, therefore add richness to the study (Malina, Norreklit, \& Selto, 2010).

\subsubsection{Contextualization}

Ellis (2014) states that quantitative research is connected to the gathering of numerical data. This type of research allows us to collect data more quickly, analyze it and discover cause-effect relations from the obtained results.

The quantitative paradigm entails a way of seeing the world and the things in it in a manner that involves being able to measure and 'prove' things. In that respect, quantitative refers to a view of the world which is amenable to quantification where the findings of research are measurable, countable or can be represented in ways which involve numbers and statistics (Ellis, 2014, p.1).

According to Cropley (2016), qualitative research contributes to the perception of the subjective nature of reality. It allows researchers to grasp the complexities of existence and the rich and meaningful ways in which they are experienced by the individuals.

Qualitative research: (...) It is based on the fundamental idea that "reality" is subjective: Every human being constructs an individual, personal view of the world on the basis of his or her specific interactions with the external world (including the people who are part of this world). As a result, much of what a person, including researchers, regards as reality actually consists of a set of impressions, inferences and opinions in the person's mind (Cropley, 2016, p.8).

In the scenario of this study, the research topic is creativity, which very strongly resides within the world of ambiguity and abstraction. Given this context, qualitative research 
contributes to the understanding, in a richer sense, of the ways in which the participants perceive the creative aspects of the generated outputs.

The distinction between qualitative and quantitative research is somewhat blurred. In the line that separates quantitative and qualitative studies there are mixed methods research designs, which combine both quantitative and qualitative components. Among mixed methods, there are many different research designs (Onwuegbuzie \& Leech, 2002; Leech $\&$ Onwuegbuzie, 2009). In the present study a traditional quantitative research design will be used: experimental research. However, a qualitative component will be added, making it possible the comparison of creativity in its more subjective aspects.

More specifically, this research aimed at understanding to what extent the use of an Artificial Intelligence algorithm determines differences in audiovisual products, leading to differences in the perception of the elements of creativity and how those differences are expressed. This was done through the use of a Style Transfer algorithm and the evaluation of creativity was made by both experts and non-experts in visual arts.

\subsubsection{Data Sources/Participants}

The participants (data sources) for the empirical research consists of: 52 experts in visual arts and 49 non-experts in the same field. Sample selection was made according to these selection criteria and on the convenience of the researcher.

The reason for the choice of this number stems from an attempt to reach an equilibrium between the validity of the study and the ability to perform the tests on time. A too short sample would produce low validity results since it could be due to specificities of the participants. On the other hand, a very large sample would be hard to carry out on time. The decision on the number of participants was made after receiving advice from two senior researchers who suggested around this number.

The reasons why those two groups of participants were considered are: a) it is expected they are different regarding the evaluation criteria of creativity in visual arts, the focus of this research; b) they constitute the public of the visual art products. Therefore, a wider range of people were the source of data and the results are more generalizable. Experts were students with a university degree whose specialty is Art. Non-experts were those whose specialty is not arts. Paired samples were selected regarding gender and age.

Furthermore, the variables "gender" and "year of birth" were also considered important for the balance between the experimental and control groups. In case these variables were unbalanced between the experimental and control groups, the possible observed differences could be the result of differences in the division of the groups regarding those variables, instead of deriving from the application of the algorithm. Accordingly, gender, age, and expertise were tested for possible differences through the application of a MannWhitney U. Despite our concern, no significant differences were observed.

The participants were divided into two groups, an experimental and a control group. The experimental group included 27 experts and 25 non-experts and the control group included 25 experts and 24 non-experts, The individuals from the experimental group watched six shared video excerpts without AI (S) and six differentiated video excerpts of videos transformed with AI (D). The individuals from the control group watched the same six 
shared video excerpts and the same six differentiated videos but without the AI transformation. The shared videos were included in order to measure possible differences in the evaluation of creativity between both groups and taking them into account if confirmed.

The differentiated videos were the same audiovisual pieces in two versions (with and without AI transformation) and allowed us to compare the differences in the evaluation of the creativity of the videos with and without the transformation employed by the algorithm.

\subsubsection{Materials and Instruments}

\subsubsection{Materials}

For this study, 12 excerpts of artistic videos, between 45 and 115 seconds long each, were screened. The videos selected were created by the Artist Nuno Barbosa. Permission for use of the videos in this research was given by the author. The videos are artistic and include visual transformations and elements of animation and design, some resembling a moving collage. The videos often depict dream-like atmospheres and have previously suffered visual transformations that have modified the colors, elements, and atmosphere of the pieces. The reason for the choice of artistic videos that were aesthetically manipulated strongly relates to the visual nature of the Style Transfer output. If the pieces were not artistic, the stylized outputted versions would more likely be considered as more creative than the inputted ones, making this a biased comparison. The selected videos correspond to the same type of short film (music videos). This was done in order to ensure that the possible differences observed in the creativity of the pieces were due to the use of the algorithm and not a result of different types of audiovisual content.

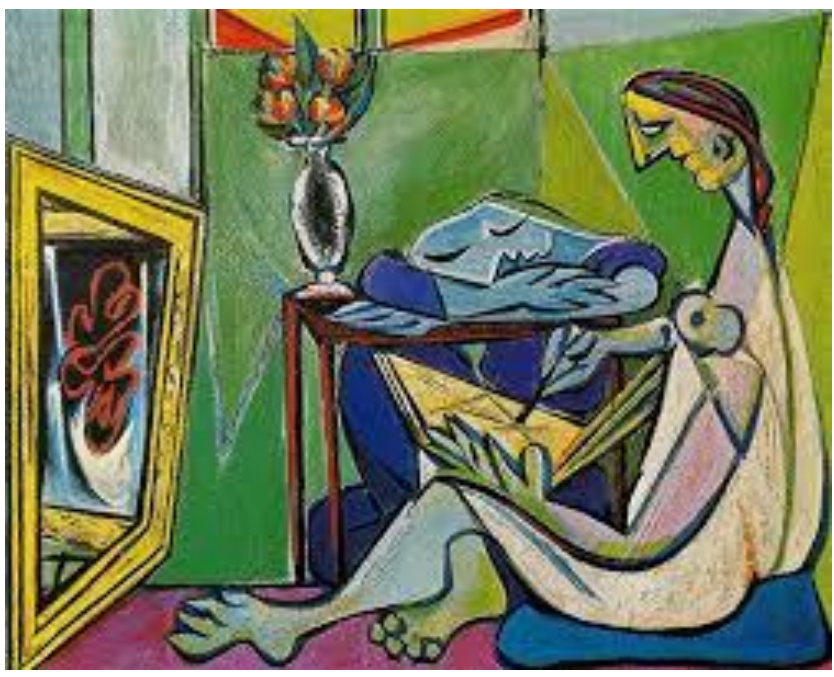

Figure 3. La Muse (Picasso, 1935).

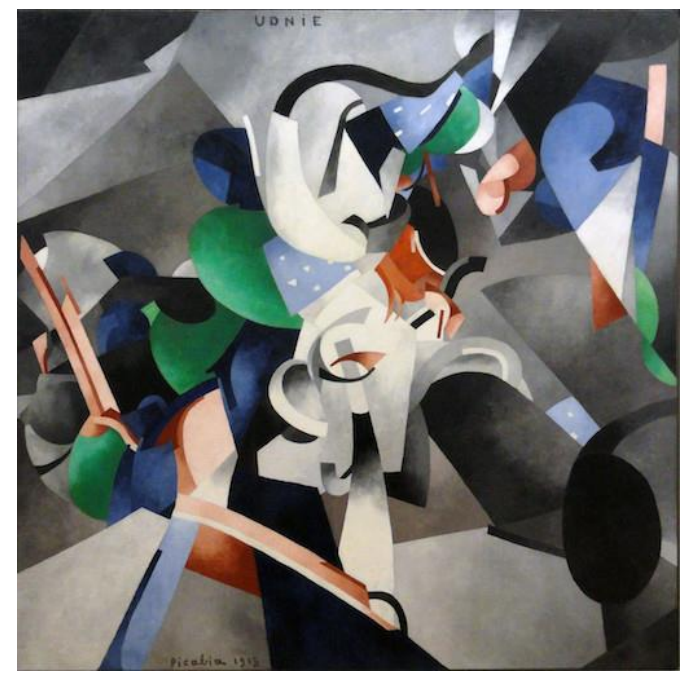

Figure 4. Undie (Picabia, 1913).

The input paintings were chosen based on two criteria: First, the pieces had to differ from each other stylistically. Secondly, they had to be pieces that were universally recognized as art pieces. Three styles were applied through the use of Style Transfer, each style was present in two videos. The styles corresponded to the following paintings: La Muse, by Pablo Picasso (1935) (see figure 3) which was applied to the videos Amplifying Creativity 
and Emmy Curl - March for Peace. The second style was the one from the painting Udnie by Francies Picabia (1913) (see figure 4) applied to the video of the song by Algodão, "Eu não sei quando te perdi" and the music video of song "Selaron" by MAGNOLIUS. The third style employed was The Great Wave off Kanagawa by Katsushika Hokusai (1831) (see figure 5), applied on the music video of Moby for the song "Be the one (Hello, Future)" and the music video of the band Junip for the song "Without you".

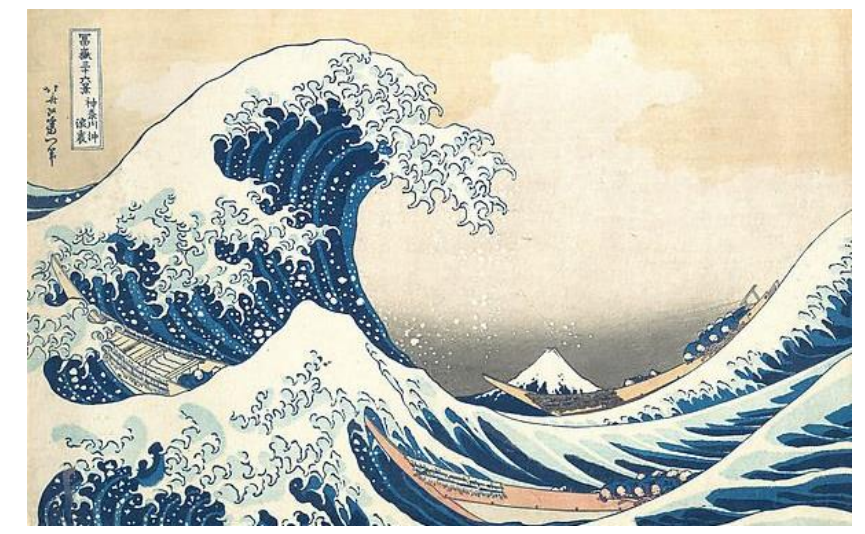

Figure 5. The Great Wave off Kanagawa (Hokusai, 1831).

To sum up, the videos were selected based on the following criteria:

a) The videos are artistic;

b) The pieces include one or more visual transformations (layering, animation, collage, projection, color treatment and other stylistic techniques);

c) The videos fit in the category of music videos;

d) The pieces include the elements of creativity as defined in the section "3.1.5.1.Dimensions of Analysis".

In regard to the style applied to each video, the choice was made based on the combination of art pieces and videos that generated visual results with less noise and visual artifacts.

\section{Video excerpts transformed through the use of Style Transfer}

Six of the twelve videos used in this research allowed us to measure the possible differences resulting from the use of the Style Transfer algorithm. As mentioned above, those videos are referred to, in this dissertation, as differentiated videos. All of the differentiated videos have a version with AI and a version without AI (see Appendix A). For each of the videos' excerpts, an encoded title was created. This was done in order to hide the true name of the video and to avoid any possible bias caused by the familiarity of the respondents with the band or influence of the title of the piece. The videos were named through the use of an 8-digit random code generator (see www.randomcodegenerator.com). In the following table the six videos chosen as differentiated videos, as well as the excerpt selected from each one of them, will be presented. 
Table 1

Videos Used in the Questionnaire (Differentiated Videos)

\begin{tabular}{lcccc}
\hline \multicolumn{1}{c}{ Video Title } & Artist & Segment & Code & Abb* $^{*}$ \\
\hline Be The One & Moby & 2:09-3:03 & zbuxf6qU & D1 \\
Eu Não Sei Quando Te & Algodão & $0: 00-0: 46$ & yjNJF6ki & D2 \\
Perdi & & & & \\
Selaron & MANGOLIUS & $2: 01-3: 02$ & 2MHnySdu & D3 \\
Amplifying Creativity & Hugo Gamelas & $0: 13-1: 22$ & aaLu9eG8 & D4 \\
Without You & Junip & $1: 40-2: 36$ & uhxb4j2q & D5 \\
March For Peace & Emmy Curl & $1: 11-1: 54$ & qqAbSsQu & D6 \\
\hline
\end{tabular}

Note. *Abbreviation.

1) The video made for the band Moby, for the song "Be The One". This audiovisual piece mixes a futuristic environment with macro-shots of the human body.

2) Another video is the one made for the singer Algodão, more specifically, for the song "Eu Não Sei Quando Te Perdi". The piece depicts dream-like atmospheres, mixing animation with shot video through the use of a green screen.

3) The music video for the song "Selaron" from the band MAGNOLIUS was also selected for the transformed video's set. The video presents 2D animated graphic elements placed on top of several buildings that metaphorically illustrate the lyrics of the song.

4) Another video that was used was Amplifying Creativity. This video presents a guitar player improvising a song while a series of 3-dimensional shapes emerge from around him and from within the guitar.

5) The music video for the song "Without You" from the band Junip, was another video that was chosen for this study. The video blends $2 \mathrm{D}$ animation and shot video illustrating the creation of life through visual metaphors in nature scenarios.

6) The music video of the song "March for Peace" by Emmy Curl was also employed for the experimental group. This video presents the artist singing in nature environments.

\section{Video excerpts without transformation}

The same criteria was used in the selection of the videos that were not transformed, the shared videos (see Appendix A). The videos in this group were seen by both the control and experimental groups and aimed at identifying possible differences in the evaluation of creativity between them. Those differences should be taken into consideration for the 
comparison between the control and experimental groups regarding AI and No-AI videos. In the following table the shared videos, as well as the segments selected from the original pieces, are presented.

Table 2

Videos Used in the Questionnaire (Shared Videos)

\begin{tabular}{lcccc}
\hline \multicolumn{1}{c}{ Video Title } & Artist & Segment & Code & Abb* \\
\hline Quattuor Elementa & $\begin{array}{c}\text { Vasco Negreiros } \\
\text { \& João Pargana }\end{array}$ & $0: 37-1: 50$ & WFmCkgPG & S1 \\
Fado Cyborg & Júlio Resende & $0: 31-1: 40$ & m7t6fBh3 & S2 \\
Come Closer & Emmy Curl & $1: 43-2: 54$ & PRftDo7P & S3 \\
Let's Go To The Moon & Júlio Resende & $0: 33-1: 42$ & Z5xZrBMm & S4 \\
Again & & & & \\
The Desert Eater & Souq & $2: 49-3: 53$ & w4epQLWx & S5 \\
Li Azul & Rouge & $1: 22-2: 24$ & tmJLMdqJ & S6 \\
\hline
\end{tabular}

Note. *Abbreviation.

1) Quattuor Elementa is one of the videos that were chosen for the experimental set. The audiovisual piece illustrates two dancers, in grayscale, dancing against a black background with a visual echo effect.

2) The video for the song "Fado Cyborg" by the artist Júlio Resende was also selected for this research. The video illustrates a musician, playing piano while a young man dances in the background, both have geometric elements projected into their bodies, creating an outer-worldly atmosphere.

3) Another audiovisual production that was chosen was the music video of the song "Come Closer" by Emmy Curl. The video is composed of visual collages, transformations and layered elements which contribute to the expressive and communicative aspects of the artwork.

4) The music video of the song "Let's Go To The Moon Again" by Júlio Resende. In the art piece different people are shown levitating in the sky in grayscale, in a slowmotion jump, while sounds of the moon landing play together with the song in the background.

5) The video for the song "The Desert Eater" from the band Souq was another piece that was selected. This video presents the story of a criminal who is hired to kill a woman and ends up being seduced by her. The shots are a mix of grayscale with reds and orange tones, creating a dark and seductive environment. 
6) Finally, the music video of the song "Li Azul" of the band Rouge was also employed for this study. The piece presents a young man, walking in the streets and singing in a theatre while he reflects upon his emotional state.

\subsubsection{Instruments}

A questionnaire was created to measure creativity, including quantitative and qualitative items. Additionally, socio-demographic questions were also included (see Appendix B).

\section{Quantitative Items}

Quantitative items were used for evaluating quantitatively the creativity of each video on a Likert scale from 1 (very low) to 5 (very high), in regard to the various aspects that compose creativity: "Aesthetic Quality", "Value of The Concept or Idea", "Communicative Power", "Expressiveness", "Originality of The Composition", and "Level of Innovation".

The reason for the choice of this scale lies within its frequent use, and consequently, a higher level of familiarity of the participants with it. The quantitative items were adapted from the questionnaire employed by Hong and Curran (2019) and considering the literature review that was previously undertaken.

\section{Qualitative item}

A qualitative question was made for qualitative evaluation of the creativity present in each video: the participants were asked to write down two words or expressions describing the video in terms of the creativity observed.

The same elements of creativity used for the quantitative items were employed for the analysis of qualitative data. Due to the qualitative nature, the items were changed from elements of measurement, to elements that contribute to the understanding of the subjective nature of the piece. Therefore, "Aesthetic Quality" was used to categorize words and terms that describe the visual and sonic characteristics of the video. "Value of the Concept or Idea" was the category in which words that describe concepts associated with the videos were inserted. "Communicative Power" included the words that related to the ideas transmitted by the artwork and the emotional impact generated by the videos in the participants. "Expressiveness" was used to aggregate adjectives that describe the elements that contribute to the communication of the ideas and emotional states conveyed in the pieces. "Originality of The Composition" included terminology that aims to describe how different and unique the pieces are. Finally, "Level of Innovation" was used to aggregate words that describe the novelty and value of the creative ideas implemented in the video.

\section{Socio-demographic Variables}

Four items focus on socio-demographic variables: gender, year of birth, educational level and professional specialty.

\subsubsection{Procedure}

The videos were uploaded to a YouTube account and hidden from the general public. The excerpts were individually cropped in the platform. The videos were unlisted so that these 
could only be accessed through the link. That procedure intended to prevent other individuals from accessing the videos in the experiment. Comments were disabled on the original page and the likes/dislikes ratio was hidden to avoid possible bias.

In the process of selection of the excerpts, particular attention was put in two specific aspects. First the level of continuity. The excerpts were cut into carefully chosen segments in order to make the process of visualization feel as uninterrupted as possible, and the piece to appear complete. Another important aspect was the exclusion of written elements, as these could influence the evaluation of creativity and make the responses to the questionnaire of the study biased.

A snowball sampling procedure was adopted. The sample was made by identifying possible subjects from the personal network of the researcher. Those subjects were contacted by email/phone number or Messenger and asked to participate in the research and to indicate other possible subjects who meet the inclusion criteria. The subjects were informed that the videos were not created by the researcher. They were asked not to share the questionnaire or its contents with non-eligible individuals. Experimental and control conditions were randomly assigned to the subjects. This procedure was stopped when the intended number of subjects was reached. In total 198 people were directly contacted by the researcher. The research protocol was composed of an introduction explaining the objectives of the study (without disclosing the use of an AI algorithm), an informed consent and six videos, each one followed by the questionnaire (see Appendix B). The email address of the subjects was provided by those subjects who would like to receive a summary of the results of the study. In total 118 individuals participated. The first round of checking the answers led us to exclude 17 participants, in order to keep the ratio regarding the socio-demographic variables balanced between both groups. The participants were selected randomly, considering the existing imbalance between the experimental and control conditions. At the end of this procedure the final sample was made up of 101 participants, 52 in the experimental group (22 males and 30 females; 27 experts and 25 non-experts; age mean: 31.48 ) and 49 in the control group (19 males and 30 females; 25 experts and 24 non-experts; age mean: 33.69 ).

The control and the experimental groups both watched 12 videos, six shared videos and six differentiated videos, which were divided by four questionnaires ( $\mathrm{K}$ and $\mathrm{L}$ for the control group, and $\mathrm{X}$ and $\mathrm{Y}$ for the experimental group). The letters were randomly assigned to the questionnaires. Each participant only watched six videos, three shared videos and three differentiated videos. This was done to keep the form at an optimal length, as watching and evaluating the 12 videos excerpts could overload the subjects. To avoid bias, an extra version of each questionnaire was created, this version presented the videos in the opposing order ( $\mathrm{Kv}, \mathrm{Lv}, \mathrm{Xv}$ and $\mathrm{Yv})$. This was done in order to avoid the bias that could exist by always presenting the same video in first place. Therefore, half of the subjects watched in first place one shared video while the other half watched first a differentiated video. All subjects watched alternately shared videos and differentiated videos. Both procedures (first place and order) jointly contribute to minimizing the order and the primacy effects.

All the twelve videos of the control group are No-AI videos, while in the experimental group, half of the videos are No-AI and the other half are AI videos. Therefore, the only difference between control and experimental groups is that the control group evaluated 12 
videos without AI transformation (six shared videos plus six differentiated videos) and the experimental group evaluated six videos without AI transformation (shared videos) and six videos with AI transformation (differentiated videos).

The participants were asked to perform the rating task independently. For the quantitative analysis, the subjects were requested to evaluate each video regarding several aspects that define creative products, on a scale of 1 to 5 . For the qualitative analysis, they were asked to write two words that describe the creativity present in each video.

\subsubsection{Data Treatment and Dimensions of Analysis}

\subsubsection{Dimensions of Analysis}

A model of important concepts for the conceptualization of this study was conceived in order to clarify the relevant aspects to be explored in the empirical research (see Table 3). As mentioned above, the various elements evaluated were taken from the definitions and concepts explored in the theoretical framework and in the questionnaire used by Hong and Curran (2019) for measuring the creativity observed in the pieces made by humans and intelligent systems. The items "Experimentation of Risk Taking" and "Development of Personal Style" from the original questionnaire were not applied to this study due to focusing on creative aspects which relate to the artistic process, and are better analyzed through the study of one's progress and personal development instead of the final product.

In front of each item, a small sentence clarifying it was written in order to make the questionnaire accessible for those who are not familiar with the terminology, which was important due to the inclusion of non-experts as respondents. This clarification was also used to make the questions more objective, which contributed to ensuring that all the participants interpreted the items similarly.

Table 3

Dimensions of Creativity

\begin{tabular}{|c|c|c|}
\hline Concept & Dimension & Indicators \\
\hline \multirow{4}{*}{ Creativity } & \multirow{3}{*}{ Value of the creation } & Aesthetic quality \\
\cline { 3 - 3 } & & Value of the concepts or idea \\
\cline { 3 - 3 } & & Communicative power \\
\cline { 3 - 3 } & \multirow{2}{*}{ Originality of the product } & Originality of the composition \\
\cline { 3 - 3 } & & Level of innovation \\
\hline
\end{tabular}

The dimensions chosen for the analysis of the concept of creativity were defined based some of the authors and concepts introduced in the theoretical framework, such as: a) the object of analysis (the creative product) defined by Amabile (1982); b) the importance of communication in multimedia products as introduced by Dolese (2015); c) the definitions of creativity presented by Sarkar and Chakrabarti (2015); d) the concept of originality as 
defined by Runco and Jaeger (2012); e) the definition of expressiveness introduced by Osborne (1982); and f) the concept of creative process as defined by Taylor (2017).

According to Amabile, a product can be considered creative when it is evaluated as creative by those familiar with the domain in which it was created. In this study audiovisual pieces are the object of analysis, therefore the dimensions "Value of The Creation" and "Originality of The Product" were considered relevant. The first one relates to the core aspects of creative products in which the piece might be considered more or less valuable, and is measured through the indicators "Aesthetic Quality", "Value of The Concept or Idea", "Communicative Power" and "Expressiveness". The second one refers to how novel and innovative the piece is and was measured through the indicators "Originality of The Composition" and "Level of Innovation". In the next paragraphs, the various indicators, the reason for the choice of each one as well as the sources of inspiration for their creation will be presented.

\section{Aesthetic Quality}

The first element of the questionnaire asks participants to evaluate the aesthetic quality of the artwork. This element was considered pertinent to the study due to contributing to the measuring of the impact of the visual transformations that are employed by the algorithm in the visual quality of the piece. The source of inspiration for this item was the element "Aesthetic Value" present in the questionnaire by Hong and Curran (2019). Next to it, the following clarifying sentence is presented "I consider that the various elements of the composition are properly constructed and visually interconnected".

\section{Value of The Concept or Idea}

The proper communication of ideas and concepts is crucial for multimedia production, namely in artistic products (Dolese, 2015). Therefore, it is relevant to measure the impact of AI systems in the perception of the concepts and ideas present in the videos. This item was also inspired by the ideas presented by Sarkar and Chakrabarti (2015), who define the creation of ideas as an important part of the creative process. The element was further clarified through the use of the sentence "I consider that the idea or concept is rich and profound" in the questionnaire.

\section{Communicative Power}

As stated previously, it is crucial to communicate properly through multimedia products. Furthermore, not only the quality of the message is relevant, the strength of the communicative ability is also important. Therefore, this indicator was selected for the study, due to measuring one of the core aspects of multimedia products, the ability to communicate emotions and ideas. The element "Communicative Power" was also based on the questionnaire by Hong and Curran (2019). The original item present in this questionnaire was described as "Successful Communication of Ideas". For the present study this was changed to "Communicative Power" due to the terminology transmitting better the intended idea in the Portuguese language. Next to the item, it was written the following clarification: "I consider that a message was transmitted". 


\section{Expressiveness}

Expressiveness relates to the elements that contribute to the communication of emotional states Osborne (1982). This item was considered relevant for the analysis of the possible differences originated by the use of Style Transfer. This indicator was inspired by the item "Degree of Expression" present in the questionnaire. In front of this element the following clarification was made "I consider that the elements are organized in a way that contributes to the communication of the message".

\section{Originality of The Composition}

The fifth element that the participants were asked to rate was the originality of the composition. As stated Runco and Jaeger (2012) originality is one of the key aspects of creativity. Therefore, this item was chosen to measure the impact of the AI algorithm on the originality of the pieces. This item was also inspired by the element "Composition" present in the questionnaire that served as a reference. The word "originality" was added in order to further communicate the intent of the question. The sentence "I consider that the composition is outside of the norm", was written next to the item in order to further explain it.

\section{Level of Innovation}

The sixth element aimed to contribute to the evaluation of how "Innovative" the pieces were. According to Taylor (2017), Innovation relates to the creation of something novel that positively impacts the world. Therefore, this item was considered as important to the understanding how the AI algorithm impacts the originality of the creative product. This was also inspired by the item "Degree of Improvement and Growth" from the questionnaire and the ideas of Sarkar and Chakrabarti (2015), previously presented. This element was clarified through the use of the sentence: "I consider that I saw something new".

\subsubsection{Quantitative Data treatment}

The quantitative evaluation of the six short videos without AI intervention made by the experimental group was compared with that made by the control group. It was expected to find no significant differences between the experimental group and the control group.

The quantitative evaluations of the six short videos with AI intervention (experimental group) and without AI intervention (control group) were compared. It was expected to find significant differences (higher creativity in the AI intervention short videos). In order to treat the data a Mann-Whitney U test was applied through the use of the software SPSS.

\subsubsection{Qualitative Data treatment}

A content analysis of the words describing the videos was performed. The words used by the experimental group were compared with the words used by the control group about the shared videos, which aimed at verifying if they were similar. Following this, the corresponding comparison is performed regarding the videos which have two versions (AI and No-AI). This process was made using Nvivo software, version 12. The data was classified according to six main categories adapted from the concepts expressed in the 
quantitative items. Additionally, a category "Others" was added for responses that were outside of the scope of the question, such as: "I don't know what term means".

Two researchers coded the responses considering that each word or expression must be coded in only one category. Where differences in coding occurred, they discussed until agreement was reached. In an attempt to attain a higher level of accuracy, researchers from both psychology and arts were involved in the coding process. 


\section{Results}

\subsection{Data Analysis}

\subsubsection{Quantitative Data}

First, the elements of creativity of the shared videos were tested for significant differences between experimental and control groups. A Mann-Whitney U test was performed. This test was chosen to identify significant differences in the ordinal variables (elements of creativity) between both categorical groups categorical (AI/No-AI). No significant differences were observed between control and experimental groups regarding the overall results. In the analysis of that comparison one video at a time, only one significant difference in the evaluation of creativity (out of 36) was found. That difference refers to the element "Originality of The Composition" of the video S1, being the score given by the experimental group higher than that given by the control group. We consider that this result means that the experimental and control groups are equivalent regarding the criteria that contributes to the judgement of the creativity of videos. For this reason, the comparison experimental vs control of the evaluation of the differentiated videos seems to be a consequence of the characteristics of the audiovisual pieces (see Appendix C).

Concerning the differentiated videos, which have an AI version and a corresponding NoAI version, some significant differences were found. Three dimensions were rated significantly higher in the experimental group and four dimensions were evaluated significantly higher in the control group. Three of the six differentiated videos did not show any significant differences between experimental and control groups. One video (D1) was rated significantly higher in four of the six elements of creativity by the control group. Another video (D2) was rated significantly higher in three of the six elements of creativity by the experimental group. There was also a mixed result (D6), as one video was rated significantly higher in two of the six elements of creativity by the experimental group and one element by the control group (see Table 4).

Table 4

Results Mann-Whitney U Test Elements of Creativity for each Differentiated Video

\begin{tabular}{lcccccccc}
\hline & \multicolumn{7}{c}{ Mean Rank } & \multicolumn{2}{c}{ Sum Ranks } & & & \\
\cline { 2 - 5 } \multicolumn{1}{c}{ Construct } & Cont. & Exp. & Cont. & Exp. & MWU & Z & p \\
\hline Video D1 & & & & & & & \\
Aesthetic & 31.65 & 19.12 & 728 & 497 & 146 & -3.207 & $.001^{* *}$ \\
Concept & 31.00 & 19.69 & 713 & 512 & 161 & -2.916 & $.004^{* *}$ \\
Communication & 30.22 & 20.38 & 695 & 530 & 179 & -.411 & $.011^{*}$ \\
Expressiveness & 30.33 & 20.29 & 697.5 & 527.5 & 176.5 & -2.633 & $.008^{* *}$ \\
Originality & 24.15 & 25.75 & 555.5 & 669.5 & 279.5 & -2.530 & .681 \\
$\quad$ Innovation & 22.54 & 27.17 & 518.5 & 706.5 & 242.5 & -1.217 & .224 \\
Video D2 & & & & & & & \\
$\quad$ Aesthetic & 23.46 & 26.37 & 539.5 & 685.5 & 263.5 & -.752 & .452
\end{tabular}




\begin{tabular}{|c|c|c|c|c|c|c|c|}
\hline \multirow[b]{2}{*}{ Construct } & \multicolumn{2}{|c|}{ Mean Rank } & \multicolumn{2}{|c|}{ Sum Ranks } & \multirow[b]{2}{*}{ MWU } & \multirow[b]{2}{*}{$\mathrm{Z}$} & \multirow[b]{2}{*}{$\mathrm{p}$} \\
\hline & Cont. & Exp. & Cont. & Exp. & & & \\
\hline Communication & 22.00 & 27.65 & 506 & 719 & 230 & -1.441 & .150 \\
\hline Concept & 20.67 & 28.83 & 475.5 & 749.5 & 199.5 & -2.115 & $.034 * *$ \\
\hline Expressiveness & 22.70 & 27.04 & 522 & 703 & 246 & -1.441 & .257 \\
\hline Originality & 18.41 & 30.83 & 423.5 & 801.5 & 147.5 & -2.115 & $.001 * *$ \\
\hline Innovation & 19.04 & 30.27 & 738 & 787 & 162 & -3.237 & $.004 * *$ \\
\hline \multicolumn{8}{|l|}{ Video D3 } \\
\hline Aesthetic & 25.69 & 27.31 & 668 & 710 & 317 & -.409 & .682 \\
\hline Concept & 26.50 & 26.50 & 689 & 689 & 338 & .000 & 1.000 \\
\hline Communication & 26.92 & 26.08 & 700 & 678 & 327 & -.211 & .833 \\
\hline Expressiveness & 24.88 & 28.12 & 647 & 731 & 296 & -.808 & .419 \\
\hline Originality & 25.08 & 27.92 & 652 & 726 & 301 & -.706 & .480 \\
\hline Innovation & 25.12 & 27.88 & 653 & 725 & 320 & -.681 & .496 \\
\hline \multicolumn{8}{|l|}{ Video D4 } \\
\hline Aesthetic & 26.23 & 26.77 & 682 & 696 & 331 & -.137 & .891 \\
\hline Concept & 25.54 & 27.46 & 664 & 714 & 313 & -.476 & .634 \\
\hline Communication & 26.81 & 26.19 & 697 & 681 & 330 & -.153 & .878 \\
\hline Expressiveness & 26.79 & 26.21 & 696.5 & 681.5 & 330.5 & -.147 & .883 \\
\hline Originality & 25.46 & 27.54 & 662 & 716 & 311 & -.522 & .602 \\
\hline Innovation & 26.19 & 26.81 & 681 & 697 & 330 & -.152 & .879 \\
\hline \multicolumn{8}{|l|}{ Video D5 } \\
\hline Aesthetic & 28.33 & 24.67 & 736.5 & 641.5 & 290.5 & -.933 & .351 \\
\hline Concept & 28.31 & 24.69 & 736 & 642 & 291 & -.916 & .360 \\
\hline Communication & 30.31 & 22.69 & 788 & 590 & 239 & -1.902 & .057 \\
\hline Expressiveness & 28.81 & 24.19 & 749 & 629 & 278 & -1.153 & .249 \\
\hline Originality & 24.96 & 28.04 & 649 & 729 & 298 & -.771 & .441 \\
\hline Innovation & 25.46 & 27.54 & 662 & 716 & 311 & -.517 & .605 \\
\hline \multicolumn{8}{|l|}{ Video D6 } \\
\hline Aesthetic & 31.11 & 19.60 & 715.5 & 509.5 & 158.5 & -3.057 & $.002 * *$ \\
\hline Concept & 21.89 & 27.75 & 503.5 & 721.5 & 227.5 & -1.538 & .124 \\
\hline Communication & 25.24 & 24.79 & 580.5 & 644.5 & 293.5 & -.116 & .908 \\
\hline Expressiveness & 24.76 & 25.21 & 569.5 & 655.5 & 293.5 & -.119 & .905 \\
\hline Originality & 18.54 & 30.71 & 426.5 & 798.5 & 150.5 & -3.116 & $.002 * *$ \\
\hline Innovation & 18.37 & 30.87 & 422.5 & 802.5 & 146.5 & -3.202 & $.001 * *$ \\
\hline
\end{tabular}

Note. Cont.$=$ Control group $;$ Exp. $=$ Experimental group; $M W U=$ Mann-Whitney U test statistics; $Z=Z$-score $(M W U) ; p=$ Significance $(2$-tailed); $* p<.05 ; * * p<.01$.

\subsubsection{Qualitative Data}

Each of the participants was requested to qualify the creativity of the videos using two words or expressions. In total 1208 references were coded following the previously described process. In the category "Others" 20 references were coded. In "Aesthetic 
Quality" 453 references were coded, in "Communicative Power" 329, "Value of The Concept or Idea", 239, "Expressiveness", 61, "Level of Innovation". 27 and "Originality of The Composition", 79. The category "Others" refers to references that were excluded from the analysis, due to not being real responses to the question. For instance, answers such as "I don't know" or just a comma were considered no responses. Regarding the shared videos, eight references from the experimental group and four from the control group were excluded for the indicated reasons. Regarding the differentiated videos, six from the experimental and two from the control group were excluded for the same reasons.

Considering the differences between control and experimental conditions, shared videos and differentiated videos have been separated. For the shared videos, the qualitative analysis was used to see if there was an equivalence in the criteria used for the qualitative evaluation of creativity between both the control and experimental groups.

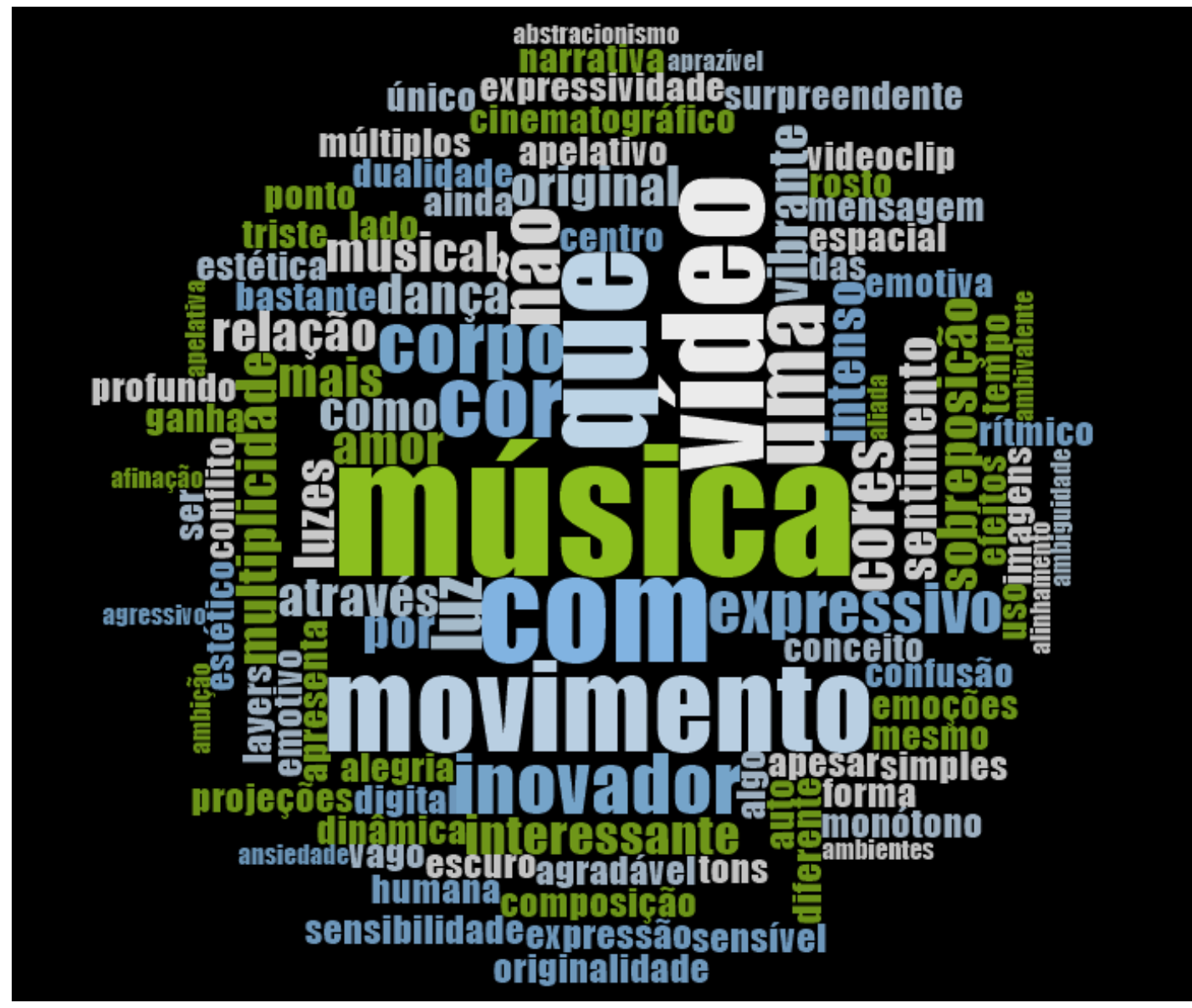

Figure 6. Word Cloud of The Shared Videos of the Control Group. 


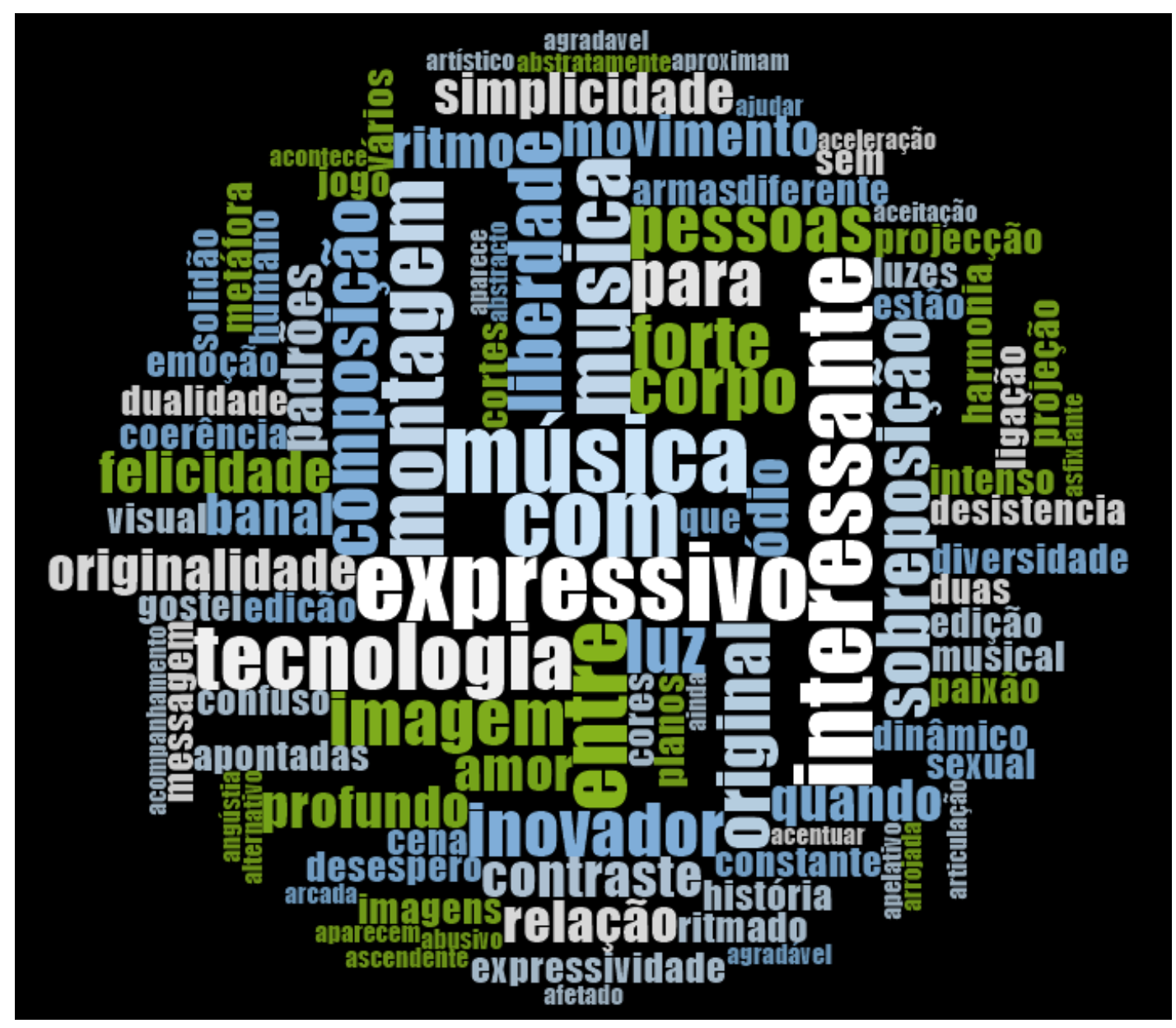

Figure 7. Word Cloud of The Shared Videos of the Experimental group.

A list was made of the most used terms by both groups. Similar terms such as "Color" and "Colors" were aggregated. It was observed that, in general, most of those terms were used with a similar frequency to evaluate both groups (see figures 6 and 7). Moreover all the 10 most frequent were used by both groups. The term "Music" was used 11 times in the control group and nine times in the experimental group. The word "Color" was employed nine times in the control group and three in the experimental group. The word "Light" was referred five times in the control group and seven times in the experimental group. The term "Movement" was employed eight times in the control group and three in the experimental group. The term "Video" was mentioned eight times in the control group and one time in the experimental group. "Image" was mentioned two times in the control group and six times in the experimental group. The word "Overlap" was used three times in the control group and four times in the experimental group. The term "Montage" was used one time in the control group and five times in the experimental group. Finally, the word "Body" was employed two times in the control group and three times in the experimental group. For further details see Table 5. 
Table 5

Word Counts for Control and Experimental Groups

\begin{tabular}{|c|c|c|c|}
\hline Word & Control & Experimental & Total \\
\hline Music & 11 & 9 & 20 \\
\hline Color & 9 & 3 & 12 \\
\hline Light & 5 & 7 & 12 \\
\hline Movement & 8 & 3 & 11 \\
\hline Video & 8 & 1 & 9 \\
\hline Image & 2 & 6 & 8 \\
\hline Overlap & 3 & 4 & 7 \\
\hline Montage & 1 & 5 & 6 \\
\hline Body & 2 & 3 & 5 \\
\hline Dance & 3 & 1 & 4 \\
\hline Contrast & 1 & 3 & 4 \\
\hline Vibrant & 3 & 0 & 3 \\
\hline
\end{tabular}

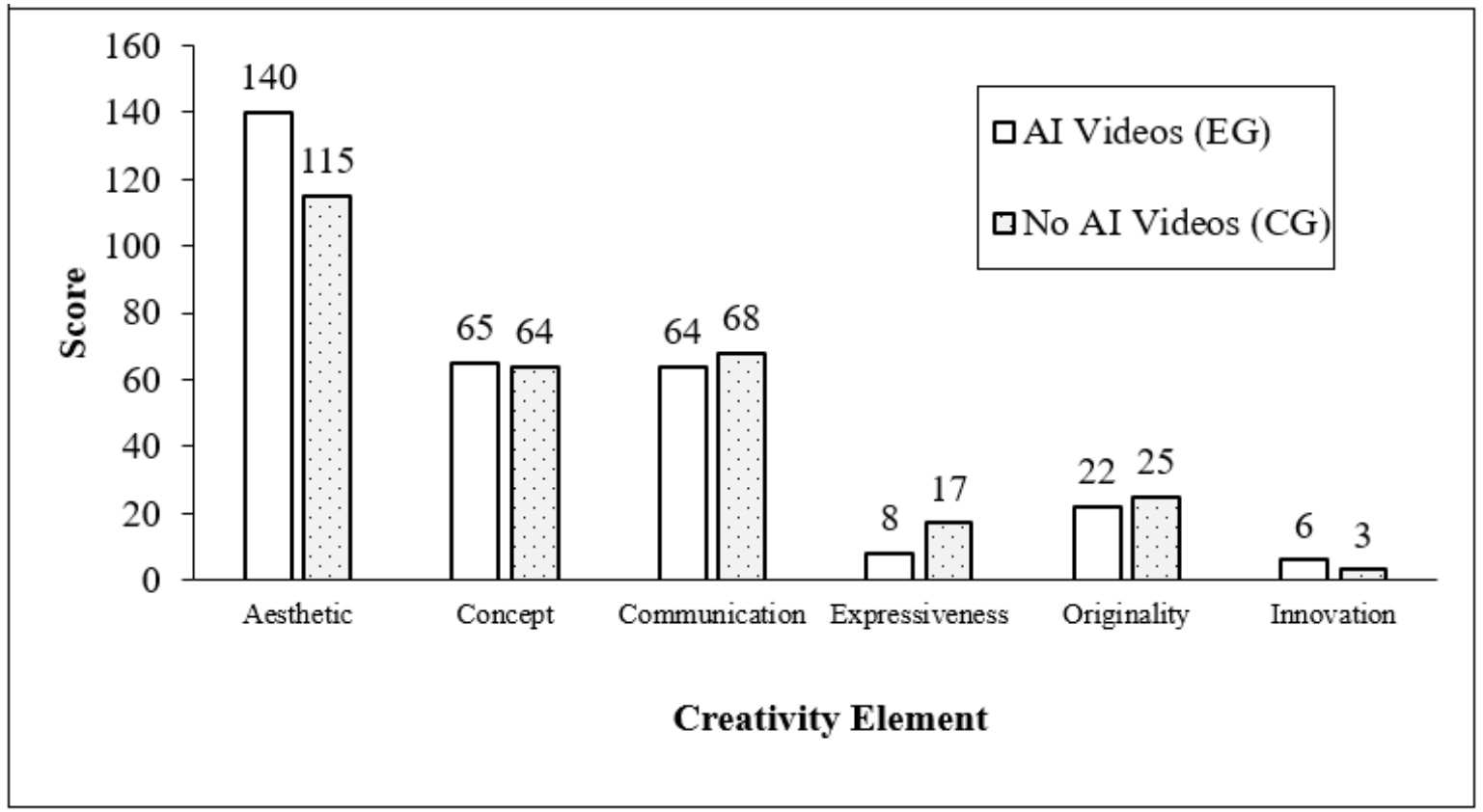

Figure 8. Scores on Creativity Elements for AI and No-AI Videos.

In the differentiated videos, a total, 305 references were coded for the experimental group and 293 for the control group. As shown in figure 8, the most coded element for both groups was "Aesthetic Quality". "Level of Innovation" was the least coded element of creativity for both groups. Table 6 shows the frequency of responses related to each element of creativity per video. 
Table 6

Qualitative Content per Video (Differentiated Videos)

\begin{tabular}{|c|c|c|c|c|c|c|c|}
\hline \multirow[b]{2}{*}{ Video } & \multirow[b]{2}{*}{$\begin{array}{l}\text { Content of } \\
\text { Creativity }\end{array}$} & \multicolumn{6}{|c|}{ Elements of Creativity } \\
\hline & & Aesthetic & $\begin{array}{l}\text { Commu } \\
\text { nication }\end{array}$ & Concept & $\begin{array}{l}\text { Express } \\
\text { iveness }\end{array}$ & $\begin{array}{c}\text { Innovati } \\
\text { on }\end{array}$ & $\begin{array}{c}\text { Original } \\
\text { ity }\end{array}$ \\
\hline \multicolumn{8}{|l|}{ AI Video } \\
\hline D1 & 52 & 24 & 10 & 13 & 1 & 1 & 3 \\
\hline D2 & 51 & 23 & 15 & 7 & 2 & 1 & 3 \\
\hline D3 & 50 & 18 & 7 & 18 & 0 & 2 & 5 \\
\hline D4 & 50 & 31 & 11 & 5 & 1 & 0 & 2 \\
\hline D5 & 50 & 18 & 11 & 13 & 4 & 1 & 3 \\
\hline D6 & 52 & 26 & 10 & 9 & 0 & 1 & 6 \\
\hline Total & 305 & 140 & 64 & 65 & 8 & 6 & 22 \\
\hline \multicolumn{8}{|l|}{$\begin{array}{l}\text { Non-AI } \\
\text { Video }\end{array}$} \\
\hline D1 & 46 & 9 & 12 & 16 & 5 & 0 & 4 \\
\hline D2 & 45 & 17 & 14 & 7 & 3 & 0 & 4 \\
\hline D3 & 52 & 27 & 7 & 10 & 2 & 2 & 4 \\
\hline D4 & 52 & 28 & 10 & 9 & 3 & 0 & 2 \\
\hline D5 & 52 & 14 & 14 & 16 & 2 & 1 & 5 \\
\hline D6 & 45 & 20 & 11 & 6 & 2 & 0 & 6 \\
\hline Total & 292 & 115 & 68 & 64 & 17 & 3 & 25 \\
\hline
\end{tabular}

Note. AI Video = experimental group; Non-AI Video = control group.

Regarding the differentiated videos, since there are two versions (AI and No-AI) of each video, the differences and commonalities of each pair were selected for analysis. Below are present at least two of the most cited words of each category. For the videos D1, the terms in the category "Aesthetic Quality" differed for both groups. While in the AI video, the aesthetic characteristics are described through a variety of words such as "Effects", "Montage" "Impressionism" and "Abstract". In the No-AI videos, the words seem to describe the more technical aspects of the image, such as "Colors", "Technique", "Photography" and "Rhythm". For the category "Value of The Concept or Idea" both groups described the concepts "Children", "Life", "Nature" and terms that relate to the experience of memories and past experiences. A difference found in the evaluation of both videos was that in the No-AI version words relating to commercial use such as "Advertisement", "Institutional" and "Marketing" were employed. In the category "Communicative Power" some differences were observed. For the No-AI video, terms related to affective states such as "Nostalgia", "Emotional" and "Profound" were used. For the AI videos, "Nostalgia" was used as well, but more often terms such as "Confusing" and 
"Strange" were used to describe the piece. For the element of creativity "Expressiveness" there were differences in the responses of the participants. The AI video had one response with the word "Expressive". The No-AI video had a wider variety of responses, including the terms "Personality", "Focus" and "Revealing". In the category "Originality of The Composition", the responses were not widely different for both groups. The AI video was evaluated both positively, with the terms "Original" and "Out of This World", and negatively, with the term "Average". The No-AI video also had positive evaluations of originality, seen in the use of words such as "Diverse" and negative ones that categorize it as "Common". Regarding the category "Innovation", only the AI video had a response, the word "Rupture".

In the videos D2, for the category "Aesthetic Quality", both the AI and No-AI versions included the terms "Effects" and "Music". The AI video also had responses that differed from the No-AI version, with words that refer to a stylistic approach, such as "Texture", "Impressionism" and "Overlap". The word "Cohesive" was also used to describe the piece. In the No-AI version, often terminology that refers to the tones of the video was used, through words such as "Black and White" and "Dark". Terms that connect with drawing were also often employed, through words such as "Illustration" and "Animation". For the category "Value of The Concept or Idea", the concepts associated with the AI version were mostly abstract, including terms such as "Symbolic", "Fragmented Word" and "Unfolding". The words used to describe the concept of the No-AI video were less abstract, often deriving from direct associations made with specific elements on the screen or the typology of the video. Words such as "Night", "Storytelling", and "Virtual Reality" were included in this section. In the category "Communicative Power" some differences were also observed. While both groups included terminology related to the emotional experience of sadness that's transmitted by the singer, in the AI version this was more frequent, through the use of words such as "Pain", "Fear" and Regret". For the No-AI version, responses also included a negative emotional response of the participants to the video, seen in the use of terms like "Abominable" and "Exaggerated". In regard to the category "Expressiveness", the AI video was described through the use of the term "Expressive" repeatedly. For the No-AI video, there was a wider variety in the terms used to describe them, which included words like "Poetic" and "Objective". For the category "Originality of The Composition", the AI and No-AI videos both had a similar amount of negative evaluations of creativity, observed in the use of words such as "Okay" and "Forgettable" for the AI video and, "Basic" and "Cliche" for the No-AI video. The videos also both had one positive evaluation of creativity each, for the AI version, the word "Originality" was used and for the No-AI version the term "Imagination" was employed. Finally, only the AI video had one response for the category "Level of Innovation", which was the term "Innovative" to describe the creativity of the piece.

For the videos D3 the terms were similar between the AI and No-AI videos. For the "Aesthetic Quality" words such as "Colorful" and "Dynamic" were employed to describe both videos. The term "Excessively Filled" was also used to describe the AI pieces. The No-AI video was described as "Random" and "Disconnected". For the category "Value of The Concept or Idea", the videos were, once again described by some similar terms, such as "Urban". Furthermore, the word "Art" was employed for the AI video and the terms "Simple" and "Meaningless" were used to describe the No-AI video. Regarding the category "Communicative Power" the answers strongly differed between both groups. While the AI video was described as transmitting the more negative emotional states, such 
as "'Insecurity" and "Anxiety", the No-AI video was described as transmitting more positive emotions, which is seen in the use of words such as "Happy" and "Funny". For the category "Expressiveness", the AI video had no answers, and the No-AI video had the answers "Expressiveness" and "Expressivity". Once again in the category "Originality of The Composition" the AI video was classified similarly to the No-AI video, both groups include the term "Originality". The AI video was classified as "Different" and "Creative" and the No-AI version as "Singular". Finally, for "Level of Innovation", the videos were also both coded with the word "Innovative", and the No-AI video was described through the term "Transformation".

Regarding the videos D4 the experimental and control groups evaluate similarly the aesthetic aspects of the pieces. Both versions included the words "Color" and "Rhythm". For the AI version, the terms "Movement" and "Animation" were often used. In the No-AI version, the words "Sound" and "Dynamic" were employed. For the element "Value of The Concept or Idea" a wider range of words were associated with the AI video in comparison with the No-AI video. For the AI video there were used concepts such as "Harmony" and "Fantasy". For the No-AI video words such as "Simplicity" and "Abstraction" were used. On "Communicative Power" some differences were observed, as the No-AI version seemed to be perceived as more emotionally impactful, here words such as "Energetic", "Pleasant" and "Intense" were used to describe the piece. Furthermore, terms such as "Funny", "Captivating" and "Calm" were employed for the AI version. In the category "Expressiveness", for the No-AI video, merely three responses were used. Here, the use of everyday objects as elements that contribute to the creation of the song was highlighted and the words "Expressivity" and "Translation" were included. For the AI video only one answer was noted, the word "Expression". In the category "Originality of The Composition" the words used by both groups were similar. In the No-AI video, the term "Imaginative" was used twice, for the AI video the words "Different" and "Imagination" were used to describe the piece. Concerning the category "Level of Innovation", no words were used to describe the videos D4.

For the videos D5, of the category "Aesthetic Quality" the most used words were "Movement" and "Color" (No-AI) and "Music" and "Visual effects" (AI). For the category "Value of The Concept or Idea" some of the words used were similar in both groups. "Nature" and "Life" (No-AI) and "Nature" and "Free" (AI). For this pair of videos, it is relevant to highlight that in the No-AI version, more varied words related to an abstract description of the video, such as "Symbolic" and "Surreal" were used to describe the composition. In regard to the category "Communicative Power" there were some differences in the evaluation of both videos. In the AI video the answers include serene and strong emotional states, with words such as "Calm" and "Intense". In this AI video, we also see some descriptions of dream-like states, with words such as "Magical" and "Dreamlike". In the No-AI video, words such as "Peace" and "Serenity" that relate a calm emotional state are also present in the participant's descriptions. In this version of the video, we also see terms that describe a strong emotional state, such as "Intense" and "Shock", but there are no words describing oneiric states. For the "Expressiveness" category the answers to both versions of the video were similar and included the words "Focus" and "Expressiveness". The two only noticeable differences were the presence of the term "Computerized Language" and "Metaphor" to describe the expression of the AI video. In the category "Originality of the Composition" both videos were described similarly, sharing the word "Different" and other terms that describe the creativity of the 
pieces, "Picturesque" (AI) and "Imagination" (No-AI). For the category "Level of Innovation" the same happened, with the AI video being described as "Novel", and the NoAI version as "Innovative".

The videos D6 had some differences in the qualitative evaluations of the element of creativity "Aesthetic Quality". The word "Color" was often used to describe both videos. The terms: "Abstract", "Impressionism", "Textured" and "Effects" were used for the AI video. The word "Image Noise" was also used to describe the AI video. For the No-AI version, the terms "Light", "Effects" and "Empty" were used. In regard to the "Value of The Concept or Idea", the concepts used to describe both videos varied strongly. For the No-AI video, terms that connect with an altered state of perception such as "Alternative", "Shifted Reality", "Parallel Universe" and "Exploration" were employed. On the other hand, for the No-AI version, the concepts used mostly described materialized elements, present on the video, such as "Cat" and "Bicycle", with the exception of the use of the concept "Simple". For the category "Communicative Power" both groups evaluated the video through the words "Interesting", "Freedom" and "Lightweight". For the AI video words related to hallucinatory experiences were included, such as "Psychedelic" and "Trip". In the No-AI video, more terms related to the affective experience, such as "Sensible" and "Caring" were added. In the creativity element "Expressiveness", only the No-AI videos were rated in the expressivity of the pieces, through the use of the words "Spontaneous" and "Expressive". Regarding the category "Originality of The Composition" there were some differences in the words used to describe the videos. In the AI video, some terms that evaluated creativity negatively such as "Average" and "Common" were used. The same happens for the No-AI video, in which we see the repeated use of the term "Cliche" and words such as "Basic". Furthermore, for the AI video, more frequently terms that positively describe the creativity of the piece were used, such as "Original" and "Different", while for the No-AI video, only one term that describes creativity positively, the word "Special", was employed. Finally, In the "Level of Innovation" element, only the AI video was classified as such in the qualitative evaluation, through the use of the word "Innovative".

In general, qualitative differences between the AI and No-AI versions of the videos were found. Those differences express the transformation made by the use of Style Transfer. Some of those differences are positive such as for the category "Value of The Concept or Idea" of the AI video D5, in which more diverse and abstract terms were used to describe the concepts of the video. Similarly, in the same category of the video D6, we observe a wider use of abstract terms like "Shifted Reality" and "Parallel Universe". Some of the differences derived from the use of the algorithm are negative, such as in the category "Aesthetic Quality", of the video D1 (AI) that was described as "Confusing" and "Strange" and the video D3, which in the same category was described as being excessively filled.

\subsection{Discussion}

The results of this study show that the implementation of this algorithm can higher the evaluation of the elements of creativity, as it was the case with the videos D2 and D6. Style Transfer can also negatively impact the public's perception of creativity, as it was the case in the parameter "Aesthetic Quality" of the videos D1 and D6 and the evaluation of "Value of The Concept or Idea", "Communicative Power" and "Expressiveness" of the video D1. However, as observed in the videos D3, D4 and D5, in some scenarios, the use of this 
algorithm can also have no significant impact on the public's perception of creativity. But why do these scores differ? What impacts Style Transfer's success or failure as a technique for enhancement of creativity in videos? A number of aspects might play a role in the disparity among the evaluation of the different videos. In the next paragraphs each of the videos will be analyzed and possible reasons for differences in the score of the various elements of creativity will be considered.

Regarding the video D2, the employment of Style Transfer was beneficial for the rating of the video in terms of "Concept", "Originality" and "Innovation". The first element might have been impacted by the change in the environment employed by the Style Transfer. The original video consists of green screen shooting mixed with graphic elements. Style Transfer makes the video more visually consistent and takes away from the feeling of "out of place" that can sometimes emerge when applying a green screen video to digitallycreated environments. This contributes to an enhancement in the public's appreciation of the richness of the idea and concept. Style Transfer's contributions to a higher evaluation in the categories "Originality of The Composition" and "Level of Innovation", might be due to two reasons. First, the fact this is a novel technique, that is not often employed in videos, secondly its ability to enhance certain visual elements of the video. The style changes the shapes in a way that can contribute to the creation of an interesting and novel aesthetic. In this excerpt, the employed style impacted the several graphic elements giving them more definition and creating new shapes around them. While the original video is more cinematic, the second one resembles a storybook as the colors become more vibrant and the elements more like objects of a fantasy world, this contributes to a change not only in the concept of the video, but also in the perceived creativity. The tree branches, mountains and moon, part of the scenario, were aesthetically redefined acquiring the looks of a painting and enhancing the environment and emotional impact of the piece.

Regarding the video D6, it is important to consider that the original excerpt of this piece was more visually clean than most of the other videos, which might have made it as if the implementation of Style Transfer denoted the video of a higher visual richness. In most scenes, the algorithm created shapes very distinct from those existing in the original video, contributing to higher ratings in the categories "Originality of The Composition" and "Level of Innovation", just as it happened in the video D2. As previously stated, the fact that Style Transfer is a novel technique can also contribute to the high scores in "Level of Innovation". Furthermore, each output will be influenced by the inputted style, which can be very unique, and this can affect positively the evaluation of the categories "Originality of The Composition" and "Level of Innovation" of the transformed pieces. However, in this video, the overall quality of the image was affected, as the Style Transfer failed to properly employ the style in a way that creates a clear distinction between shapes in areas with less contrast making the images at times unclear. Another aspect that was an issue in this excerpt was the ways in which light variation and shots with blur affected the temporal consistency of the image which resulted in visual noise in some scenes. Consequently, the element "Aesthetic Quality" was rated significantly higher in the control group.

The previously mentioned changes could also negatively impact the perception of creativity of videos, as it was the case for the video D1. In this piece, the Style Transfer algorithm took away from the "Aesthetic Quality" and "Expressiveness" of the video. This might be a consequence of the style employed, which faded the colors, contrasts and the depth of the image. In future applications it will be important to properly manage all these 
aspects in order to create a piece that is positively influenced by the use of the algorithm. Furthermore, some parts of the video included previous visual transformations, textures and layering of images, the employment of the chosen style negatively impacted those elements making them imperceptible. The video aims to present memories, therefore the variation in colors and textures that emerge in the piece is important to the understanding of the concept. By applying this particular style (from the painting The Great Wave off Kanagawa by Katsushika Hokusai, 1831), through the Style Transfer algorithm, those elements lose definition. Therefore, important aspects that contribute to the communication of the story are lost, and the concept becomes unclear. These previously presented aspects are thought to have affected the communication of ideas and the public's perception of the concepts negatively in the AI piece. What about the videos that did not show significant differences in the elements that define creativity? In the next paragraphs each of those videos will be presented and the results will be considered.

In regard to the video D3, this could be due to the fact that in this piece, a series of graphic elements are composed on top of buildings, interacting with them and contrasting the rich colors of the designed elements with the textures and neutral tones of the buildings. In the context of the Style Transfer algorithm, this does not appear to bring anything particularly novel to the composition. On one hand it contributes to the creation of an interesting aesthetic, on the other hand it takes away from the feeling of two different coexisting elements, making the tones, textures and lines more uniform.

The same appears to happen in the videos D4 and D5. Both of these audiovisual creations included shot and digitally designed objects. Here, the contrast between the shot video and the $3 \mathrm{D}$ and $2 \mathrm{D}$ animated elements is lost. Contrary to the video $\mathrm{D} 2$, in which the AI version had significantly higher ratings in three of the elements of creativity brought by the uniformization of the video, in D4 and D5 the contrast was intentional and contributed to a richness in the aesthetic of the pieces. Furthermore, before the transformation, the digitally produced elements had colors that differed from the rest of the scenario, which contributed to them standing out and enhanced the contrast between the various objects in the picture. By applying the Style Transfer algorithm, this difference was erased. Just as in the previous video, this AI system added a new visual environment and contributed to the creation of a new aesthetic, while simultaneously removing some aspects that influence the video's creativity, therefore not producing any significant higher or lower values in the evaluation of the elements of creativity.

Therefore, it appears that when the algorithm is applied to videos that intentionally contrast graphic elements with shot video to create a visually diverse environment, there are no significant differences in terms of the creativity ratings. On the other hand, in videos in which the goal is to create an immersive environment and to make the elements in the image uniform, such as in D2, the audiovisual piece is positively affected by the use of this algorithm.

Regarding the qualitative data results show also, that similarities and differences were found between the experimental and control groups. Taken together, qualitative and quantitative results show that the AI system, in some cases, contributes to the improvement of the creativity expressed in the videos, as it was the case for the videos D2, in the category "Communicative Power" here, the words describing the emotional states were more positive for the AI video, than for the No-AI video, in which the piece was seen as 
"Abominable" and "Exaggerated". Something similar happened in the category "Value of The Concept or Idea" of the videos D6. Here, the application of the algorithm caused the participants to use terminology that is connected with an altered state of perception instead of terms that simply describe the objects observed in the AI video as it happened with the No-AI version.

In other cases, the application of the algorithm seems to negatively impact the quality of creativity, as it was the case in the category "Communicative Power" of the video D1. Here, terms such as "Strange" and "Confusing" were used to describe the pieces. Furthermore, in the category "Expressiveness" of the same video a wider variety of responses were given for the No-AI video. The research hypothesis of this project was that the transformation through the use of the Style Transfer algorithm would make the pieces be perceived as more creative. However, it seems that AI systems (in this case, Style Transfer), per se, are not sufficient to improve the creativity expressed in the videos, but maybe need the artist's intervention instead. That complex influence highlights the importance of the concept of Hybrid Intelligence (Dellermann et al., 2019). As previously mentioned, humans and machines have complementary skills. Therefore, this concept consists of the convergence of Artificial Intelligence and Human Intelligence, which can unite these different strengths in a way that benefits the world.

\subsection{Limitations and Future Studies}

One limitation for this study consists of the fact that we only study one artificial intelligence algorithm, Style Transfer created by Jin (2018), which means our results only apply to that algorithm. In the future it will be important that similar studies are conducted with other AI systems, in order to consolidate the obtained results.

Creativity is one of the characteristics of the videos and the way they are perceived. Other aspects must be considered in future studies. For example, the preference, the beauty, and the appropriateness of the videos to specific objectives and contexts.

Furthermore, the transformation produced by the algorithm was introduced on top of a finalized art piece. In the future, it will be important to conduct studies in which the Style Transfer is applied during the creative process. This limitation encourages future studies. Also, the study focuses solely on art videos, other art forms should also be explored in future studies, equivalent to the one here presented. Another limitation stems from the fact that the study was conducted online due to the pandemic, which made difficult a tight experimental monitoring of the data collection.

Despite those limitations this study is expected to contribute to the understanding of the impact of the use of Artificial Intelligence systems in arts and entertainment. Human beings appear not to be replaceable by the algorithm. The new avenues that appear by the use of those systems seem to be promising. 


\section{Conclusion}

The present dissertation aimed: a) to verify to what extent the use of an Artificial Intelligence algorithm ensures a more creative artistic outcome in videos as perceived by individuals; b) to identify what are the creative elements that the use of an Artificial Intelligence algorithm improves or deteriorates in an artistic product, the videos; c) to describe the perception by the spectators regarding the pieces created with and without the use of an Artificial Intelligence algorithm.

It was highlighted that AI is a growing field of research and practice. Several AI software and algorithms were described, such as the ones created by Gatys et al. (2016), Johnson et al. (2016), Ulyanov et al. (2017), Ruder et al. (2016) and Elgammal et al. (2017). The AI Style transfer algorithm composed by Jin (2018) was introduced as the one used in the study.

Theoretical approaches for creativity were described in the section "2. Theoretical Framework". Here, the elements of creativity were presented as defined by Walia (2019), Sarkar and Chakrabarti (2015) and Runco and Jaeger (2012). Following this, the creative agent as introduced by Sternberg (2018) is presented. Next, the concept of creative process by Amabile (1996) and Taylor (2017) are introduced and computational creativity is defined. Finally, this section is completed through the exploration of the empirical studies that relate Artificial intelligence to creative production conducted by Elgammal et al. (2017), Hong and Curran (2019), and Chen et al. (2019). These studies showed while AI systems appear to be able to generate some of the perceived attributes of creativity that humans produce they appear not to be able to generate artworks with the same level of creativity (Elgammal et al., 2017; Hong \& Curran, 2019).

Next, the method was presented, an experimental study which makes use of both qualitative and quantitative data and both types of data were contextualized in the present research. Next, the participants (52) experts and (49) non-experts were described, and the criteria for their choice was explained. After this, the chosen videos were presented, as well as the excerpts used in the experimental study and the styles applied on each transformed video. In the next part, the instruments used for qualitative and quantitative data collection are introduced, together with the elements of creativity "Aesthetic Quality", "Value of The Concept or Idea", "Communicative Power", "Expressiveness", "Originality of The Composition", and "Level of Innovation".

There were both an experimental and a control group. Twelve videos were used in the research. Six shared videos were used to identify differences in the measurement of creativity of both groups and six differentiated videos were used to measure differences in creativity generated by the software. The shared videos were the same for both groups. The differentiated videos were the same six videos but transformed by the AI algorithm for the experimental group and without AI transformation for the control group.

Three videos showed significant differences between experimental and control groups. For the quantitative data, firstly, the video (D1) was evaluated significantly higher by the control group in four out of the six elements of creativity. Secondly, the video (D2) was evaluated significantly higher by the experimental group in three of the six elements of 
creativity. Thirdly, the video (D6), was evaluated significantly higher in two of the six elements of creativity by the experimental group and in one element by the control group. Regarding the qualitative data, there were also some differences in the evaluation of creativity between the control and experimental groups. However, those differences between conditions do not have negative or positive meanings only present in one of both groups. Therefore, they are just qualitative differences which combined with the quantitative results emphasize that the effect of AÍ is not necessarily negative or positive. Consequently, human management of AÍ use in artistic creations gains relevance in the present study.

It can be concluded that the undertaken study suggests that: a) for some videos there seems to occur an improvement in the elements of creativity originated by the implementation of the Style Transfer software; b) in other videos the opposite occurs. Our results reinforce the idea that it is important that this system is mediated by artists, reinforcing the concept of Hybrid Intelligence presented by Dellermann et al. (2019).

In the past, the introduction of new technologies in the art sphere caused some transformations in the field, such as the photographic camera. Likewise, it is expected that artificial intelligence will deeply transform the artistic world (Arcas, 2017). AI systems have already been shown to help in the process of ideation, as presented in the study by Chen et al. (2019), which is an important task for artistic creation.

Since humans and machines have complementary skills (Dellermann et al., 2019), these technologies might become useful tools for artists, allowing them to transform pieces in ways that would be impossible or very time-consuming without them. Furthermore, AI systems do not appear to originate a creative result on their own, but to benefit from the presence of humans. In this study, the Style Transfer was not used as a tool to serve the creative product but instead applied with neutral research purposes. The results showed that by doing this the evaluation of creativity varied irregularly, sometimes positively, others negatively. Therefore, the artist does not appear to be replaceable, as their central position during the creation of art pieces is reinforced by this study. The obtained results seem to indicate that the concept of hybrid intelligence will be a promising path for the application of these technologies. In future research the use of such algorithms by artists can help verify to what extent these systems can contribute to an improvement of the creativity of the products when used as a tool during the creative process.

The limitations of the present study encourage further research that can contribute to the study of other AI systems, exploring elements such as the preference, the beauty, and the appropriateness of the videos and that analyze the use of Artificial Intelligence systems during the creative process. Despite those limitations, the goals of the present study were reached as it can be witnessed throughout this dissertation. 


\section{References}

Amabile, T. M. (1982). Social psychology of creativity: A consensual assessment technique. Journal of Personality and Social Psychology, 43(5), 997-1013. Retrieved from https://www.apa.org/pubs/journals/psp/

Amabile, T. M. (1996). Creativity in context: Update to "The Social Psychology of Creativity". New York: Westview Press.

Arcas, B. A. (2017). Art in the Age of Machine Intelligence. Arts, 6(4), 18. doi:https://doi.org/10.3390/arts6040018

Audry, S., \& Ippolito, J. (2019). Can Artificial Intelligence Make Art without Artists? Ask the Viewer. Arts (2076-0752), 8(1), 35. doi:https://doi.org/10.3390/arts8010035

Avdeeff, M. (2019, October 11). Artificial Intelligence \& Popular Music: SKYGGE, Flow Machines, and the Audio Uncanny Valley. Arts. 8(4), 130. doi:https://doi.org/10.3390/arts8040130

Bonini, T., \& Donoughue, P. (2017, August 11). Artificial intelligence and creativity: If robots can make art, what's left for us? Retrieved from https://www. abc.net.au/news/2017-08-11/artificial-intelligence-can-ai-be-creative/8793906

Brownlee, J. (2019, October 26). Difference Between a Batch and an Epoch in a Neural Network. [Web log post]. Retrieved from https://machinelearningmastery.com/difference-between-a-batch- and-an-epoch/

Chen, L., Wang, P., Dong, H., Shi. F., Han, J., Guo, Y., Childs, P., Xiao, J., Wu, C. (2019). An artificial intelligence based data-driven approach for design ideation. Journal of Visual Communication and Image Representation, 61, 10-22. doi:https://doi.org/10.1016/j.jvcir.2019.02.009

Cohen, Harold (2010). Orcas Center, Crossroads Lecture Series [Lecture]. Retrieved from http://www.aaronshome.com/aaron/publications/orcastalk2s.pdf

Colton, S., López de Mántaras, R., \& Stock, O. (2009). Computational Creativity: Coming of Age. AI Magazine, 30(3), 11-14. doi:https://doi.org/10.1609/aimag.v30i3.2257

Cropley, A. J. (2016). Qualitative research methods: A practice-oriented introduction for students of psychology and education. Riga, Latvia: Zinātne.

DeepAI. (n.d.-a). What is NumPy?. Retrieved from https://www.educba.com/what-isnumpy/

DeepAI. (n.d.-b) Per-Pixel Loss Functions. Retrieved from https://deepai.org/machinelearning-glossary-and-terms/per-pixel-loss-function

DeepAI (n.d.-c). What is an Epoch? (n.d.). Retrieved from https://deepai.org/machinelearning-glossary-and-terms/epoch 
Dellermann, D., Ebel, P., Söllner., M., Leimeister, J. M. (2019, March) Hybrid Intelligence. Business \& Information Systems Engineering. doi:https://doi.org/10.1007/s12599-019-00595-2

Dolese, M. J. (2015). Art as Communication: Employing Gricean Principles of Communication as a Model for Art Appreciation (Doctoral dissertation, CUNY Academic Works). Retrieved from https://academicworks.cuny.edu/gc_etds/907/

Elgammal, A., Liu, B., Elhoseiny, M., Mazzone, M., (2017, June). CAN: Creative Adversarial Networks, Generating "Art" by Learning About Styles and Deviating from Style Norms. Paper presented at the ICCC'17. 8th International Conference on Computational Creativity, USA, Atlanta.

Ellis, P. (2014). The language of research (part 1): Research paradigms. Wounds UK, 10(2), 118-119.

Garcia, C. (2016, August 23). Harold Cohen and Aaron - A 40 year collaboration. Retrieved from https://computerhistory.org/blog/harold-cohen-and-aaron-a-40-yearcollaboration/

Gatys, L. A., Ecker, A. S., \&. Bethge, M., (2016). Image Style Transfer Using Convolutional Neural Networks. Paper presented at the IEEE'16 Conference on Computer Vision and Pattern Recognition (CVPR). Paradise, NV. doi:10.1109/CVPR.2016.265

Hambeukers, D. (2018, September 14). The concept of the concept of a website. Retrieved from https://medium.com/artscientist/the-concept-of-the-concept-of-a-website$\underline{95487 \mathrm{~d} 112643}$

Hokusai, K. (1831). The Great Wave off Kanagawa. Retrieved From https://www.metmuseum.org/art/collection/search/45434

Hong, J.-W., Curran, N. (2019). Artificial Intelligence, Artists, and Art: Attitudes Toward Artwork Produced by Humans vs. Artificial Intelligence. ACM Transactions on Multimedia Computing, Communications, and Applications, 15(25), 1-16. doi:https://doi.org/10.1145/3326337

IBM. (n.d.). Data science and Machine Learning. Retrieved from https://www.ibm.com/analytics/machine-learning

Jin, C. (2018). Real-time Style Transfer [Computer software]. Retrieved from https://github.com/ChengBinJin/Real-time-style-transfer

Jing, Y., Yang, Y., Feng, Z., Ye, J., Yu, Y., \& Song, M. (2019). Neural Style Transfer: A Review. IEEE Transactions on Visualization and Computer Graphics. doi:https://doi.org/10.1109/tvcg.2019.2921336

Johnson, J., Alahi, A., Fei-Fei, L. (2016). Perceptual Losses for Real-Time Style Transfer and Super-Resolution. In: Leibe B., Matas J., Sebe N., Welling M. (Eds.), Lecture Notes in Computer Science: Vol.9906. Computer Vision - ECCV 2016 (pp.694-711). doi:https://doi.org/10.1007/978-3-319-46475-6_43 
Kok, J. N., Boers, E. J., Kosters, W. A., Putten, P. V., \& Poel, M. (2009). Artificial Intelligence: Definition, Trends, Techniques and Cases. In N. J. Kok. (Ed.). Artificial Intelligence (pp. 1-6). United Kingdom: Eolss Publishers Co. Ltd.

Kumar, C. (2018, August 31). Artificial Intelligence: Definition, Types, Examples, Technologies. Retrieved from https://medium.com/@ chethankumargn/artificialintelligence-definition-types-examples-technologies-962ea75c7b9b

Kunda, M. (2018). Visual mental imagery: A view from artificial intelligence. Cortex, 105, 155-172. doi:https://doi.org/10.1016/j.cortex.2018.01.022

Leech, N. \& Onwuegbuzie, A. (2009). A typology of mixed methods research designs. Quality and Quantity, 43(2), 265-275. doi:10.1007/s11135-007-9105-3

Lomas, A. (2018). On Hybrid Creativity. Arts, 7(3), 25. doi:https://doi.org/10.3390/arts7030025

López de Mántaras, R. (2013). Computational Creativity. ARBOR Ciencia, Pensamiento y Cultura. 189(764), 1-13. doi:hp://dx.doi.org/10.3989/arbor.2013.764n6005

Malina, M., Norreklit, H., \& Selto, F. (2010). Lessons Learned: Advantages and Disadvantages of Mixed Methods Research. Qualitative Research in Accounting \& Management, 8(1), 59-72. doi:10.1108/11766091111124702

Mazzone, M., \& Elgammal, A. (2019). Art, Creativity, and the Potential of Artificial Intelligence. Arts, 8(1), 26. doi:https://doi.org/10.3390/arts8010026

Mit media lab. (n.d.). Shelley: Human-AI Collaborated Horror Stories. Retrieved from https://www.media.mit.edu/projects/shelley/overview/

Mordvintsev, A., Olah, C., Tyka, M. (2015, June 17th). Inceptionism: Going Deeper into Neural Networks. [Web log post] Retrieved from https://ai.googleblog.com/2015/06/inceptionism-going-deeper-into-neural.html

Moura, L. (2018). Robot Art: An Interview with Leonel Moura. Arts, 7(3), 28. doi:https://doi.org/10.3390/arts7030028

Naqa, I. E., Murphy, M. J., \& Li, R. (2015). Machine Learning in Radiation Oncology: Theory and Applications. Berlim: Springer.

Natarajan, B. K. (1991). Machine Learning: A Theoretical Approach. Burlington: Morgan Kaufmann.

Nelson, D. (2019, October 20). What is Reinforcement Learning? Retrieved from https://www.unite.ai/what-is-reinforcement-learning/

Onwuegbuzie, A., \& Leech, N. L. (2002). On Becoming a Pragmatic Researcher: The Importance of Combining Quantitative and Qualitative Research Methodologies. International Journal of Social Research Methodology, 8(5), 375-387. doi:https://doi.org/10.1080/13645570500402447 
Oppy, G., \& Dowe, D., (2019). The Turing Test. Retrieved from https://plato.stanford.edu/archives/spr2019/entries/turing-test

Ornes, S. (2019, March). Science and Culture: Computers take art in new directions, challenging the meaning of "creativity". Proceedings of the National Academy of Sciences, 116(11), 4760-4763. doi:https://doi.org/10.1073/pnas.1900883116

Osborne, H. (1982). Expressiveness in the Arts. The Journal of Aesthetics and Art Criticism, 41(1), 19-26. doi:10.2307/430820

Park, Y. (2019). Can Artworks by Artificial Intelligence be Artworks? AM Journal of Art and Media Studies, 20, 113-121. doi:http://dx.doi.org/10.25038/am.v0i20.332

Picabia, F. (1913). Undie. Retrieved from http://www.iamkon.com/projects/007_ml_style_transfer_p5/index.html

Picasso, P. (1935). La Muse. Retrieved from https://www.wikiart.org/en/pablo-picasso/amuse-1935

Prince, S. J. (2012). Computer vision: models, learning and inference. United Kingdom: Cambridge University Press.

Rayner, A. (2016). Can Google's Deep Dream become an art machine? Retrieved from https://www.theguardian.com/artanddesign/2016/mar/28/google-deep-dream-art

Ruder, M., Dosovitskiy, A., \& Brox, T. (2018) Artistic Style Transfer for Videos and Spherical Images. International Journal of Computer Vision, 126(11), 1199-1219. doi:10.1007/s11263-018-1089-z

Runco, M. A., \& Jaeger, G. J. (2012). The Standard Definition of Creativity. Creativity Research Journal, 24(1), 92-96. doi:10.1080/10400419.2012.650092

Saha, S. (2018, December 15). A Comprehensive Guide to Convolutional Neural Networks — the ELI5 way. [Web log post]. Retrieved from https://towardsdatascience.com/acomprehensive-guide-to-convolutional-neural-networks-the-eli5-way-3bd2b1164a53

Sarkar, P., \& Chakrabarti, A. (2015). Creativity: Generic definition, tests, factors and methods. International Journal of Design Sciences and Technology, 21(1), 7-37. Retrieved from https://www.researchgate.net/publication/281117849_Creativity_Generic_Definition_T ests_Factors_and_Methods

SAS. (n.d.). Data mining: What it is and why it matters. Retrieved from https://www.sas.com/en_us/insights/analytics/data-mining.html

Shalev-Shwartz, S., \& Ben-David, S. (2014). Understanding Machine Learning: From Theory to Algorithms. United Kingdom : Cambridge University Press.

Sharma, S (2017). Epoch vs Batch Size vs Iterations. Retrived from https://towardsdatascience.com/epoch-vs-iterations-vs-batch-size-4dfb9c7ce9c9 
Smolic, A., Sikora, T., Ohm, J-R. (1999). Long-term global motion estimation and its application for sprite coding, content description, and segmentation. IEEE Transactions on Circuits and Systems for Video Technology, 9(8), 1227 - 1242. doi:10.1109/76.809158

Statt, N. (2017). Google's AI Duet lets you make music with a virtual pianist. Retrieved from https://www.theverge.com/2017/2/16/14641462/google-ai-duet-experiment-musicart-software

Sternberg, R. J. (2018). A triangular theory of creativity. Psychology of Aesthetics, Creativity, and the Arts, 12(1), 50-67. https://doi.org/10.1037/aca0000095

Sternberg, R. J. (n.d.). [The Investment Theory of Creativity and the Propulsion Theory of Creative Contributions]. Retrieved from http://www.robertjsternberg.com/investmenttheory-of-creativity

Szeliski, R. (2011). Computer Vision: Algorithms and Applications. United Kingdom: Springer London Ltd.

Taylor, S. P. (2017). What is innovation? A study of the definitions, academic models and applicability of innovation to an example of social housing in England. Open Journal of Social Sciences, 5(1), 128-146. Retrieved from http://insight.cumbria.ac.uk/id/eprint/3475/

Ulyanov, D., Lebedev, V., Vedaldi, A., Lempitsky, V.S., (2016). Texture Networks: FeedForward Synthesis of Textures and Stylized Images. Proceedings of the 33rd International Conference on International Conference on Machine Learning, 48, 13491357. doi:https://dl.acm.org/doi/10.5555/3045390.3045533

Ulyanov, D., Vedaldi, A., Lempitsky, V. (2017). Improved Texture Networks: Maximizing Quality and Diversity in Feed-Forward Stylization and Texture Synthesis. 2017 IEEE Conference on Computer Vision and Pattern Recognition (CVPR) (pp.4105-4113). doi:https://doi.org/10.1109/cvpr.2017.437

Urooj, W. (2019, November 26th). SciPy Tutorial: What is Python SciPy and How to use it? [Web log post]. Retrieved from https://www.edureka.co/blog/scipy-tutorial/

Walia, C. (2019). A Dynamic Definition of Creativity. Creativity Research Journal, 31(3), 237-247.

Wang, Y. (2009). On Abstract Intelligence: Toward a Unifying Theory of Natural, Artificial, Machinable, and Computational Intelligence. International Journal of Software Science and Computational Intelligence, 1(1), 1-17. doi: 10.4018/jssci.2009010101

Wilson, A. (2019, September 29th). A Brief Introduction to Supervised Learning. Retrieved from https://towardsdatascience.com/a-brief-introduction-to-supervisedlearning-54a3e3932590 
Yegulalp, S. (2019, June 18th). What is TensorFlow? The Machine Learning library explained. Retrieved from https://www.infoworld.com/article/3278008/what-istensorflow-the-machine-learning-library-explained.html

Zulko. (2017). MoviePy. Retrieved from https://zulko.github.io/moviepy/ 


\section{Appendix A}

\section{Shared Videos}

- S1:https://www.youtube.com/watch?v=VVqsObss9Cs

- S2:https://www.youtube.com/watch?v=JvUBAUv4CQs

- S3:https://www.youtube.com/watch?v=9c43VIVLggU

- S4:https://www.youtube.com/watch?v=4i1JzDbYkgc

- S5:https://www.youtube.com/watch?v=NkTAuksYzd0

- S6:https://www.youtube.com/watch?v=lLzFABSUGnU

\section{Differentiated Videos}

- D1(AI):https://www.youtube.com/watch?v=d-ASt5msegM

- D1(No-AI): https://www.youtube.com/watch?v=Rnj-JriGlc0

- D2(AI):https://www.youtube.com/watch?v=ODgwkupFoxw

- D2(No-AI): https://www.youtube.com/watch?v=8IxNvEhmWSE

- D3(AI):https://www.youtube.com/watch?v=5gN1rEZIRuc

- D3(No-AI):https://www.youtube.com/watch?v=PC2gwxP8-t4

- D4(AI):https://www.youtube.com/watch?v=hi50g-VDISI

- D4(No-AI):https://www.youtube.com/watch?v=2mMZivUA5As

- $\mathrm{D} 5(\mathrm{AI}): \underline{\mathrm{https}: / / w w w . y o u t u b e . c o m / w a t c h ? v=\mathrm{dBsExZAu} 59 \mathrm{M}}$

- D5(No-AI):https://www.youtube.com/watch?v=MI-dfWssu6w

- D6(AI):https://www.youtube.com/watch?v=60SqntJITcI

- D6(No-AI):https://www.youtube.com/watch?v=7VKffN2NGfw 


\section{Appendix B}

Questionário

(Tempo estimado de resposta: 12 minutos) Consentimento Informado No âmbito do Mestrado em Comunicação Multimédia da Universidade de Aveiro, pretende-se estudar a criatividade na produção audiovisual. Solicitamos que responda a um pequeno conjunto de questões relativas a cada um dos seis vídeos apresentados. Será salvaguardado o anonimato e a confidencialidade dos dados recolhidos e a sua utilização para fins meramente académicos. Os participantes têm o direito de desistir a qualquer momento sem qualquer tipo de prejuízo. Se tiver interesse em conhecer os resultados do presente estudo, envie um email para anadanielaperesrebelo@ua.pt colocando em assunto "Resultados Criatividade". Estimamos que demore 12 minutos a assistir aos vídeos e a responder às questões. Qualquer dúvida pode ser esclarecida pelo número de telémovel +351 931671 434, ou pelo email anadanielaperesrebelo@ua.pt. Antecipadamente Grata, Ana Daniela Rebelo (Mestranda) Prof. Dr ${ }^{\mathrm{a}}$ Inês Guedes de Oliveira (Orientadora Científica)

Section 1

\section{1}

1e. Tomei conhecimento das condições inerentes a esta investigação e concordo em participar

$\circ$ Aceito

Section 2

2e. Sexo

○ Feminino

○ Masculino

- Outro

3e. Ano de Nascimento 
4e. Tem formação de ensino superior (Bacherlato, Licenciatura, Mestrado, Doutoramento)?
○ Sim
○ Não

5e. Área de especialidade
$\circ$ Artes
○ Outra

\section{Section 3}

Visualize o seguinte vídeo em modo de ecrã inteiro e na qualidade máxima.

Section 4

Vídeo

Por favor, utilize uma escala de 1 (muito baixo) a 5 (muito elevado) para avaliar a obra observada no que se refere aos seguintes aspectos: 
6. Nível de qualidade visual (considero que os diversos elementos da composição estão bem construídos e interligados a nível visual)
$\circ 1$
$\circ 2$
$\circ 3$
$\circ 4$
$\circ 5$

\section{7}

7. Valor da ideia ou conceito (considero que a ideia ou conceito é rica e profunda)
○ 1
○ 2
○ 3
○ 4
○ 5

\section{8}

8. Poder comunicativo (considero que foi transmitida uma mensagem)
○ 1
$\circ 2$
○ 3
○ 4
○ 5 
9. Originalidade da composição (considero que a composição rompeu com a norma)
$\circ 1$
$\circ 2$
$\circ 3$
$\circ 4$
$\circ 5$

10. Capacidade expressiva (considero que os elementos estão organizados de forma a contribuir para a comunicação da mensagem)
$\circ 1$
$\circ 2$
$\circ 3$
$\circ 4$
$\circ 5$

\section{1}

11. Nível de inovação (considero que vi algo novo)
$\circ 1$
$\circ 2$
$\circ 3$
$\circ 4$
$\circ 5$ 


\section{Appendix C}

Table C1

Results (Mann-Whitney U Test) Elements of Creativity for each Shared Video

\begin{tabular}{|c|c|c|c|c|c|c|c|}
\hline \multirow[b]{2}{*}{ Construct } & \multicolumn{2}{|c|}{ Mean Rank } & \multicolumn{2}{|c|}{ Sum Ranks } & \multirow[b]{2}{*}{ MWU } & \multirow[b]{2}{*}{$\mathrm{Z}$} & \multirow[b]{2}{*}{$\mathrm{p}$} \\
\hline & Cont. & Exp. & Cont. & Exp. & & & \\
\hline \multicolumn{8}{|l|}{ Video S1 } \\
\hline Aesthetic & 23.81 & 29.19 & 619 & 759 & 268 & -1.399 & .162 \\
\hline Concept & 25.33 & 27.67 & 658.5 & 719.5 & 307.5 & -.588 & .556 \\
\hline Communication & 25.25 & 27.75 & 656.5 & 721.5 & 305.5 & -.633 & .526 \\
\hline Expressiveness & 24.44 & 28.56 & 653.5 & 742.5 & 284.5 & -1.053 & .099 \\
\hline Originality & 21.71 & 31.29 & 564.5 & 813.5 & 213.5 & -2.395 & $.017 *$ \\
\hline Innovation & 23.15 & 29.85 & 602 & 776 & 251 & -1.651 & .099 \\
\hline \multicolumn{8}{|l|}{ Video S2 } \\
\hline Aesthetic & 25.13 & 24.88 & 578 & 647 & 296 & -.064 & .949 \\
\hline Concept & 24.85 & 25.13 & 571.5 & 653.5 & 295.5 & -.074 & .941 \\
\hline Communication & 23.3 & 26.5 & 536 & 689 & 260 & -.811 & .417 \\
\hline Expressiveness & 23.85 & 26.02 & 548.5 & 676.5 & 272.5 & -.561 & .575 \\
\hline Originality & 24.54 & 25.4 & 564.5 & 660.5 & 288.5 & -.291 & .827 \\
\hline Innovation & 24.11 & 25.79 & 554.5 & 670.5 & 278.5 & -.427 & .669 \\
\hline \multicolumn{8}{|l|}{ Video S3 } \\
\hline Aesthetic & 23.02 & 29.98 & 598.5 & 779.5 & 247.5 & -1.797 & .072 \\
\hline Concept & 24.96 & 28.04 & 649 & 729 & 298 & -.779 & .736 \\
\hline Communication & 26.38 & 26.62 & 686 & 692 & 335 & -.057 & .954 \\
\hline Expressiveness & 23.81 & 29.19 & 619 & 759 & 268 & -1.402 & .161 \\
\hline Originality & 24.50 & 28.50 & 637 & 741 & 286 & -1.012 & .312 \\
\hline Innovation & 26.40 & 26.60 & 686.5 & 691.5 & 335.5 & -0.48 & .962 \\
\hline \multicolumn{8}{|l|}{ Video S4 } \\
\hline Aesthetic & 25.85 & 24.24 & 594.5 & 630.5 & 279.5 & -.423 & 672 \\
\hline Concept & 27.35 & 22.92 & 629 & 596 & 245 & -.1 .161 & .245 \\
\hline Communication & 26.83 & 23.38 & 617 & 608 & 257 & -.888 & .374 \\
\hline Expressiveness & 25.35 & 24.96 & 568 & 657 & 291 & -.176 & .860 \\
\hline Originality & 24.54 & 25.40 & 583 & 642 & 288.5 & -.223 & .823 \\
\hline Innovation & 24.7 & 25.27 & 532.5 & 692.5 & 292 & -.146 & .884 \\
\hline \multicolumn{8}{|l|}{ Video S5 } \\
\hline Aesthetic & 23.15 & 26.63 & 535.5 & 692.5 & 256.5 & -.9451 & .347 \\
\hline Concept & 21.85 & 27.79 & 502.5 & 722.5 & 226.5 & -1.535 & .125 \\
\hline Communication & 21.96 & 27.69 & 505 & 720 & 229 & -1.513 & .130 \\
\hline Expressiveness & 24.22 & 25.69 & 557 & 668 & 281 & -.403 & 687 \\
\hline Originality & 21.76 & 27.87 & 500.5 & 724.5 & 224.5 & -1.562 & .118 \\
\hline Innovation & 22.15 & 27.52 & 509.5 & 715.5 & 233.5 & -1.370 & .171 \\
\hline
\end{tabular}




\begin{tabular}{|c|c|c|c|c|c|c|c|}
\hline \multirow[b]{2}{*}{ Construct } & \multicolumn{2}{|c|}{ Mean Rank } & \multicolumn{2}{|c|}{ Sum Ranks } & \multirow[b]{2}{*}{ MWU } & \multirow[b]{2}{*}{$\mathrm{Z}$} & \multirow[b]{2}{*}{$\mathrm{p}$} \\
\hline & Cont. & Exp. & Cont. & Exp. & & & \\
\hline \multicolumn{8}{|l|}{ Video S6 } \\
\hline Aesthetic & 24.58 & 28.42 & 639 & 739 & 288 & -.995 & .320 \\
\hline Concept & 28.87 & 24.13 & 750.5 & 627.5 & 276.5 & -1.205 & .228 \\
\hline Communication & 25.67 & 27.33 & 667.5 & 710.5 & 316.5 & -.414 & .679 \\
\hline Expressiveness & 25.35 & 27.65 & 659 & 719 & 308 & -.580 & .562 \\
\hline Originality & 28.65 & 24.35 & 745 & 633 & 282 & -1.085 & .278 \\
\hline Innovation & 28.60 & 24.40 & 743.5 & 634.5 & 283.5 & -1.040 & .298 \\
\hline
\end{tabular}

